Provided for non-commercial research and education use. Not for reproduction, distribution or commercial use.

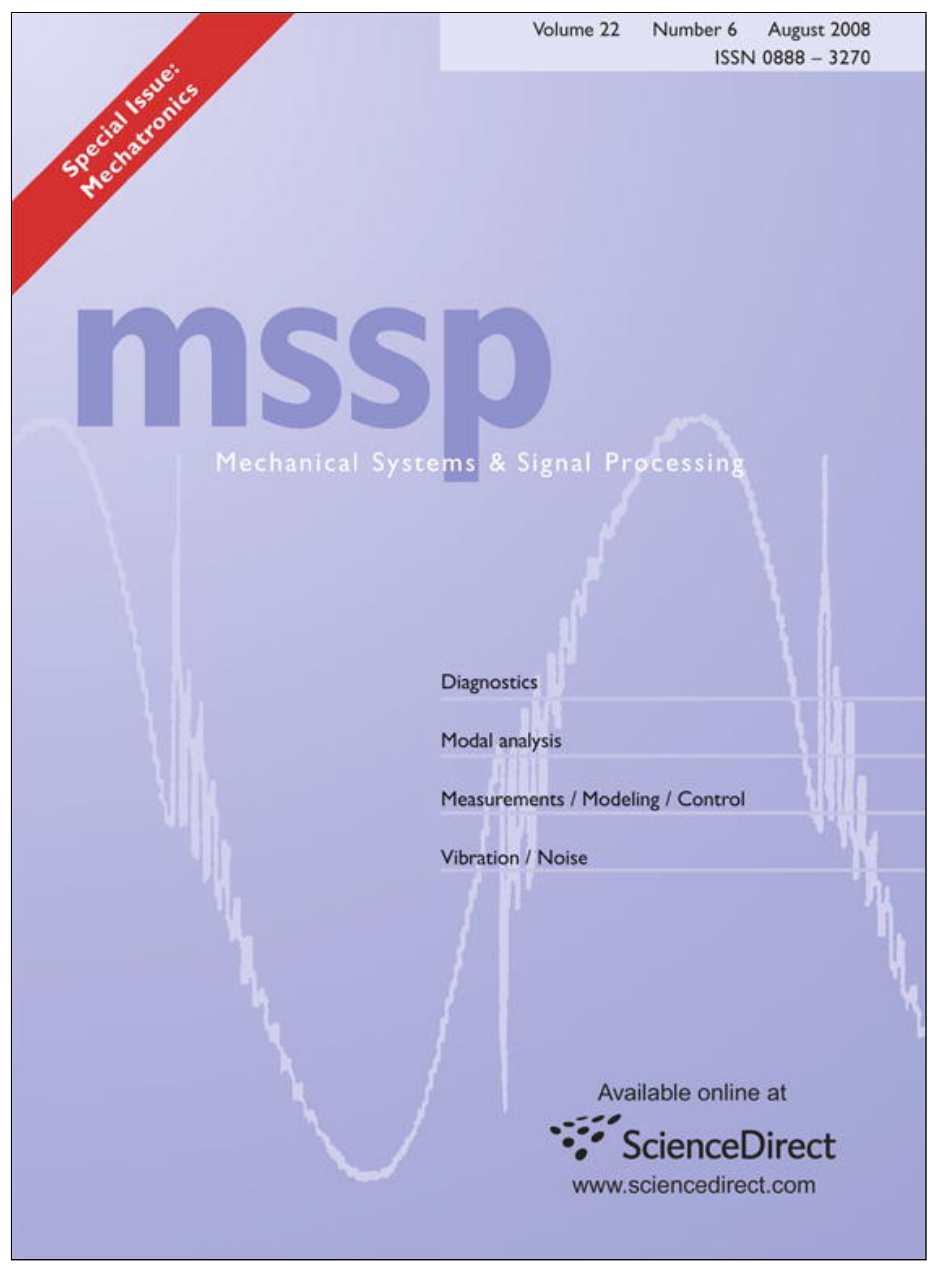

This article appeared in a journal published by Elsevier. The attached copy is furnished to the author for internal non-commercial research and education use, including for instruction at the authors institution and sharing with colleagues.

Other uses, including reproduction and distribution, or selling or licensing copies, or posting to personal, institutional or third party websites are prohibited.

In most cases authors are permitted to post their version of the article (e.g. in Word or Tex form) to their personal website or institutional repository. Authors requiring further information regarding Elsevier's archiving and manuscript policies are encouraged to visit:

http://www.elsevier.com/copyright 


\title{
On the HHT, its problems, and some solutions
}

\author{
R.T. Rato, M.D. Ortigueira*,1, A.G. Batista \\ Campus da FCT da UNL, Quinta da Torre, 2825 - 114 Monte da Caparica, Portugal \\ Received 15 December 2006; accepted 30 November 2007 \\ Available online 18 January 2008
}

\begin{abstract}
The empirical mode decomposition (EMD) is reviewed and some questions related to its effective performance are discussed. Its interpretation in terms of AM/FM modulation is done. Solutions for its drawbacks are proposed. Numerical simulations are carried out to empirically evaluate the proposed modified EMD.
\end{abstract}

(C) 2007 Elsevier Ltd. All rights reserved.

Keywords: Empirical mode decomposition; Intrinsic mode function; Parabolic interpolation; Extrema detection; Amplitude-frequency modulation

\section{Introduction}

The empirical mode decomposition (EMD) [1] is a technique to decompose a given signal into a set of elemental signals called "intrinsic mode functions" (IMFs). The EMD is the base of the so-called "Hilbert-Huang transform (HHT)" [1] that comprises the EMD and the Hilbert spectral analysis that performs a spectral analysis using the Hilbert transform (HT) followed by an instantaneous frequency computation.

The algorithm is simple and gives good results in situations where other methods fail. However, it has some drawbacks, tied with some of the assumptions needed to implement the algorithm, leading to unexpected results. There have been several attempts to solve such problems. For example, Rilling et al. [2,3] made some algorithmic variations and proposed a new stopping criterion. Besides, they gave an interpretation in terms of filter banks. They also studied the influence of sampling [4]. On the other hand, Junsheng et al. [5] studied the behaviour of the decomposition algorithm and proposed an energy difference tracking method to define a coherent stopping criterion. In another attempt [6] they use the Teager-Kaiser [7] energy operator to extract the amplitude and instantaneous frequency of a multi-component amplitude-modulated and frequencymodulated (AM/FM) signals. The problem of envelope estimation is considered by Qin and Zhong who proposed the segment power function method [8].

In this paper, we make a global appreciation of the different aspects of the algorithm, considering its drawbacks and suggesting modifications to alleviate such problems. The most important is the extrema

\footnotetext{
${ }^{*}$ Corresponding author. Tel.: + 3511 2948520; fax: + 35112957786.

E-mail addresses: rtr@uninova.pt (R.T. Rato), mdo@fct.unl.pt (M.D. Ortigueira), agb@fct.unl.pt (A.G. Batista).

${ }^{1}$ Also with INESC_ID, R. Alves Redol, 9,2 Lisbon, Portugal.
} 
determination by using a parabolic approximation and the instantaneous amplitude and frequency computation that we propose to be done through an amplitude demodulation and first-order autoregressive (AR) approximation $[9,10]$.

The paper outline is as follows. In Section 2 we describe the EMD algorithm as proposed in [1] and look inside it to understand the main difficulties and ways of avoiding them. In Section 3 we present the proposed solutions to such problems. In Section 4, we present some examples to illustrate the behaviour of the algorithm and to understand its features. Finally, we present some conclusions.

\section{The EMD}

\subsection{Outline of the EMD algorithm}

The EMD as proposed by Huang et al. [1] is a signal decomposition algorithm based on a successive removal of elemental signals: the IMFs. Given any signal, $x(t)$, the IMFs are found by an iterative procedure called sifting algorithm, which is composed of the following steps:

(a) Find all the local maxima, $M_{i}, i=1,2, \ldots$, and minima, $m_{k}, k=1,2, \ldots$, in $x(t)$.

(b) Compute the corresponding interpolating signals $M(t):=f_{M}\left(M_{i}, t\right)$, and $m(t):=f_{m}\left(m_{k}, t\right)$. These signals are the upper and lower envelopes of the signal.

(c) Let $e(t):=(M(t)+m(t)) / 2$.

(d) Subtract $e(t)$ from the signal: $x(t):=x(t)-e(t)$.

(e) Return to step (a)-stop when $x(t)$ remains nearly unchanged.

(f) Once we obtain an IMF, $\varphi(t)$, remove it from the signal $x(t):=x(t)-\varphi(t)$ and return to (a) if $x(t)$ has more than one extremum (neither a constant nor a trend).

The interpolating function is a cubic spline. By construction, the number of extrema should decrease when going from one IMF to the next, and the whole decomposition is expected to be completed with a finite number of IMFs (see [2]). We must remark that, at least conceptually, the algorithm:

- is simple;

- appears naturally;

- does not assume anything about the signal, mainly stationarity;

- can be applied to a wide class of signals.

\subsection{The EMD as an $A M / F M$ decomposition}

Consider the sifting process. The first step finds two sets of points that constitute samples of two discretetime signals. The interpolated signals give estimates of the upper and lower envelopes. If the envelopes were symmetric we would say that $x(t)$ is an AM signal [11]. The sifting procedure is an iterative way of removing the dissymmetry between the upper and lower envelopes in order to transform the original signal into an AM signal. At least conceptually, this goal would be achieved in a few steps if:

- The extrema were correctly determined.

- We had no problems with the interpolation at the extremities.

- We had no computational errors.

These difficulties will be considered later.

The above procedure allows us to conclude that each IMF is an AM signal. Moreover, as the instantaneous frequency can change from instant to instant, we can say that each IMF is an amplitude/frequency modulated (AM/FM) signal. So, the EMD is nothing else than a decomposition into a set of AM/FM modulated signals. As it is not hard to understand, the envelopes cannot vary as fast as the signal, $x(t)$. 
In spectral terms, we can say that the bandwidth of the envelopes must be a fraction of the central frequency of $x(t)$ (normally called carrier). This means that when performing the sifting we are removing the low frequency components. So, we are leaving a high frequency signal. This explains why the IMFs appear in a high to low frequency order and why the EMD is essentially a time-frequency decomposition. This also explains why the EMD behaves like a bank of filters [3]. This also explains an interesting phenomenon: if we add two IMFs with non-intercepting bands, they will be decomposed without great distortion, but if the bands intercept, they will be decomposed into a set of several IMFs. We will show this later.

\subsection{On the IMF}

For the continuous case, an abstract IMF is defined as a signal that satisfies two conditions [1]:

- In the whole signal segment, the number of extrema and the number of zero crossings must be either equal or differ at most by one.

- At any point, the mean value of the envelope defined by the local maxima and the envelope defined by the local minima is zero.

This is the original definition of IMF presented in [1]. However, the first condition is redundant unless the function at hand is discontinuous. In fact, if a function verifies the second condition, it verifies the first also, because between a peak and a valley there is always a zero, since the envelopes are symmetric.

It is not difficult to see that sinusoidal signals $\sin (2 \pi f t)$ or $\cos (2 \pi f t)$ for any real $f$ and $t$ are IMFs. It is not difficult to recognize that if $\Phi(t)$ is a continuous function, the same happens with $\sin [\Phi(t)]$ and $\cos [\Phi(t)]$. These are well-known functions in telecommunications where they are studied under the name of "angle modulation" [11]. The instantaneous frequency is, aside a constant, the derivative of $\Phi(t)$. Now, let us consider a function $g(t)=A(t) \sin [\Phi(t)]$. This is what is called "double side band" modulated sinusoid. In general, it is not an IMF, even if $A(t)$ is also a sinusoid. But, if $A(t)$ changes slowly when compared with the changes in $\sin [\varphi(t)]$, we have really an IMF. So, we can say that a function of the type $A(t) \sin [\Phi(t)]$ represents an IMF, provided that $A(t)$ is a slowly varying function. This is the so-called envelope. But we must remark that the above definition of envelope may not be a true envelope. A simple example shows this. The function $x(t)=\mathrm{e}^{-|t|} \sin (2 \pi f t)$ has $\mathrm{e}^{-|t|}$ as envelope, but almost all the extrema points of $x(t)$ do not belong to $\mathrm{e}^{-|t|}$. In some signals this fact has as consequence that the function may have segments above (below) the envelope defined by the maxima (minima). As a conjecture, we can say that the extrema envelope coincides with the "true" envelope when this has extrema locations equal to those of the signal. However, there is no simple way of defining the "true" envelope and the extrema defining envelope seems to be suitable to our objectives.

We can conclude that the definition of IMF is tied with the definition of envelope that depends also on the interpolating function used to estimate it. So, the Huang et al. [1] IMFs correspond to the cubic spline interpolator. With other interpolator we may obtain a different set of IMFs. In our simulations we also used the Akima interpolator. ${ }^{2}$ The results were very similar.

\subsection{Main drawbacks of the EMD}

Let us take a look into the above described algorithm and consider the most important steps:

- extrema locations;

- extrema interpolation;

- end effects;

- sifting stopping criterion;

- IMF removal.

\footnotetext{
${ }^{2} \mathrm{We}$ used the routine available at the MatLab site.
} 
As we have seen in the earlier last sections, the algorithm has some implicit difficulties and the procedures used in previous papers [1-3] for the above steps create several drawbacks that originate "strange" decompositions. For example, when trying to decompose a sinusoidal segment, it is expected to obtain only one IMF and no residual. This may not happen with the available algorithm implementations [1-3].

We are going to look into each step and see why it creates difficulties. We begin by the extrema computation. This does not have an obvious solution. Although most data are originated by continuous-time processes, in practice, the algorithm operates on quantized discrete-time signals. When working with this kind of signals, some special attention is necessary because the extrema may not be correctly identified. Most, if not all, of the continuous waveform actual extrema will fall in between sampling instants and will not be correctly localized. To avoid this difficulty, Rilling et al. [2] proposed the use of a fair amount of oversampling.

We have considered before the interpolation problem and the interpolation function choice. However, even if we made a reasonable choice, the effectiveness of the approximation is highly dependent on the extrema computation and may lead to some undesirable results. One of them is the "overshoots": the signal crosses the envelope. This affects the IMF estimation.

The end effects appear when we have to decide what to do with the first and last samples. The solution will affect the final decomposition:

- To consider them as maxima and minima simultaneously (this forces all the IMFs to be zero at those points).

- To consider them as maxima or minima according to the nearest extremum in order to guarantee the alternation between maxima and minima.

- To leave them free [1].

The stopping criterion is another source of problems due to its degree of arbitrariness, since it may not guarantee a total signal removal to obtain a "true" IMF.

According to the above description, the final step is the removal of the IMF from the signal. However, if the IMF is not well computed, we may be "adding" to the remaining signal a component that will appear in the following IMF. This explains partially why we do not obtain only one IMF in the case of a pure sinusoid.

\section{Some attempts to obtain a better algorithm}

\subsection{A framework for modifying the algorithm}

The EMD does not have an analytical formulation: it is based on a computational algorithm. So it performs according to its implementation details. When the same signal is fed into different EMD implementations, different results are obtained, as we will see in Section 5.1. This is confusing and inadequate. To achieve consistency and to be able to compare results, different implementations should be equivalent. Our goal is to establish a framework for EMD implementation to avoid the referred drawbacks (Section 2.4).

Based on this rationale, we state the following guiding principles to be followed in the design, implementation and checking of EMD programs:

- The IMF set obtained by multiplying a constant value to all the samples in the signal should be the IMF set of the original signal multiplied by the same constant.

- Changing the mean of the signal, it should only change the trend related IMF (the last one), leaving all the others unchanged.

- The EMD of an IMF should be the IMF itself.

- The IMF set obtained from a time reversed signal should be the time reversed IMF set of the original signal. $^{3}$

\footnotetext{
${ }^{3}$ This condition is not fundamental and can be released.
} 
These rules suggest us to:

- Remove the mean.

- Normalize the signal to a unit power.

This last procedure is important when dealing with signals with very low amplitudes as in the case of biomedical signals.

\subsection{The proposed solutions}

\subsubsection{The extrema locations}

In practice, the algorithm operates on discrete-time signals. This deserves some special attention because the extrema definition cannot be based on a continuous neighbourhood, as in calculus. It is necessary to define a classificatory function to assert whether a given sample is, or is not, an extremum. The classification of a sample as an extremum must be based on the relation of the actual sample and its left and right neighbours. Except for the first and last samples, all samples vicinity will have just those two values. Larger vicinities, with more than two values, will distress the algorithm's local feature. So, the classificatory function must receive as input just these three values. According to principle (d), commuting the preceding sample with the subsequent sample should not change a sample classification. A classification based solely on " $>$ " and " $\geqslant$ " type relations will adhere naturally to principles (b) and (c) seen in the previous section. For a given sample, $x[n]$, the used classificatory function is

\section{If $((x[n]>x[n-1])$ AND $(x[n] \geqslant x[n+1]))$}

OR $((x[n] \geqslant x[n-1])$ AND $(x[n]>x[n+1]))$ Then Return ("Is a Maximum")

Else if $((x[n]<x[n-1])$ AND $(x[n] \leqslant x[n+1]))$

OR $((x[n] \leqslant x[n-1])$ AND $(x[n]<x[n+1]))$ Then Return ("Is a Minimum")

Else Return ("Not an extrema");

However, the envelope fitting depends on the extrema accuracy (position and value). As referred above, dealing with a discrete-time signal produces an error in the location of an extremum that can be equal to half the sampling interval. To alleviate this problem, we interpolate the signal in the interval defined by the two neighbours to obtain a better extremum location and individualization [4]. Classic interpolation for band limited signals relies on the computationally demanding sinc function as the interpolation kernel. This has the disadvantage of needing a lot of samples on the right and on the left.

In agreement with our comments in Section 2.3, we are expecting that the IMFs have some degree of smoothness, mainly near the extrema. In particular, they may also have a null derivative at the extrema. This means that, near the extrema, it can be approximated by a parabola. So, parabolic interpolation comes into sight as a practical compromise between no interpolations at all and sinc based interpolations, for better location of the extrema values. Using parabolic interpolation, each extremum is estimated from only the above defined three samples. A new interpolated extremum sample is obtained, usually with no integer abscissa, and this new sample is fed into the EMD algorithm, replacing the integer abscissa extremum sample as an envelope defining point. To see what happens, assume the situation where we have an extremum near time $t=n$, and let $y(1)=x[n-1], y(2)=x[n]$, and $y(3)=x[n+1]$. The interpolating parabola is defined by $y(k)=a \cdot k^{2}+b$. $k+c(k=1,2,3)$. We have

$$
\left[\begin{array}{l}
y(1) \\
y(2) \\
y(3)
\end{array}\right]=\left[\begin{array}{lll}
1 & 1 & 1 \\
4 & 2 & 1 \\
9 & 3 & 1
\end{array}\right]\left[\begin{array}{l}
a \\
b \\
c
\end{array}\right] \text { and }\left[\begin{array}{l}
a \\
b \\
c
\end{array}\right]=\left[\begin{array}{ccc}
1 / 2 & -1 & 1 / 2 \\
-5 / 2 & 4 & -3 / 2 \\
3 & -3 & 1
\end{array}\right]\left[\begin{array}{l}
y(1) \\
y(2) \\
y(3)
\end{array}\right] .
$$

The sign of " $a$ " will allow us to decide which kind of extremum we have: $a>0$, it is a minimum; $a<0$, it is a maximum. If $a=0$ it is not an extremum. From simple geometric considerations, the coordinates $\left(t_{p}, x_{p}\right)$ for 
the extremum obtained from the interpolating parabola will be

$$
t_{p}=-\frac{b}{2 a}, \quad y_{p}=-\frac{b}{2} t_{p}+c .
$$

If $1.5<t_{p}<2.5$, we will have an extremum with a true location point at $t=n-2+t_{p}$; otherwise, there will not be an extremum.

As simple reasoning shows, this procedure gets round the use of the above classificatory function. In fact, we can run the interpolation algorithm just described for all the triplets $x[n-1], x[n]$, and $x[n+1]$ formed with the samples of the signal. The first and last samples were not considered, leaving them as not extrema. This option was taken according to the procedure described in the next section.

\subsubsection{The end effects}

In Section 2.4 we referred the end effects. These appear due to the fact that a given interpolator may not be a good extrapolator (see Fig. 1). In fact, unless we consider the initial and final points as maximum and minimum simultaneously, we have at least one extremum in each side that must be free. This means that the envelope near that point will be obtained by extrapolation. This extrapolation is poorer according to the distance to the first (last) extremum. To be more concrete, let us assume that the first extremum calculated as described in the previous section is a maximum. Then, the first point must be a minimum. So, we are not constraining the upper envelope estimator near the first point to be an extrapolator. This gives a poor result. To avoid this problem, several attempts were made such as repetition or reflection of the signal. The results were not encouraging, because we were really extrapolating the signal. Instead we decided to extrapolate the maxima and the minima. This has an important effect: the envelope estimator always behaves as an interpolator. As we will see later the end effects are almost invisible. Let us consider the beginning of the signal that we assume to be at time $t=0$. The extrapolation of the extrema as done using the following procedure:

(a) Find the first maximum, $M_{1}$, and minimum, $m_{1}$, and their time locations, $T_{1}$ and $t_{1}$. Assume, for example, that $T_{1}>t_{1}$.

(b) Insert a new maximum, $M_{0}=M_{1}$, located at $T_{0}=-t_{1}$, and a new minimum, $m_{0}=m_{1}$, located a $t_{0}=-T_{1}$.

To the end of the signal the extrapolation is similar. This procedure is correct in the face of our comments in Section 2.3: the envelope is a low pass signal in face of the original signal.

\subsubsection{The mean envelope removal}

This is an important aspect of the algorithm, since we may be adding a non-existing component that can distort the actual IMF and will appear in, at least, one of the following IMFs. To attenuate this we modified step (d) in Section 2.1 by introducing a step size $0<\alpha<1: x(t):=x(t)-\alpha \cdot e(t)$. This increases the iteration time duration, but the algorithm becomes more reliable. In principle, the parameter $\alpha$ can be arbitrary, but we found it better to choose it according to an acceptable criterion: minimize the energy of the resulting signal. This criterion leads to $\alpha$ equal to the correlation coefficient between $x(t)$ and $e(t)$.

\subsubsection{The stopping criterion}

To state a stopping criterion in the sifting procedure, we will define a resolution factor by the ratio between the energy of the signal at the beginning of the sifting, $x(t)$, and the energy of average of the envelopes, $e(t)$.

If this ratio grows above the allowed resolution, then the IMF computation must stop. This criterion gives a scale independent stopping way, as opposed to criteria based on iteration count. Only the practice and the particular problem can give a good insight into the resolution to be used. In some experiments with filtered ECG we used $40 \mathrm{~dB}$, but in the analysis of EEG we used a resolution around $50 \mathrm{~dB}$.

A useful property of the resolution factor is that it enables the researcher to set the number of IMFs. Reducing the resolution factor reduces the number of obtained IMFs. This has important practical applications, in situations where different signals should be decomposed into the same IMF number in order 
a
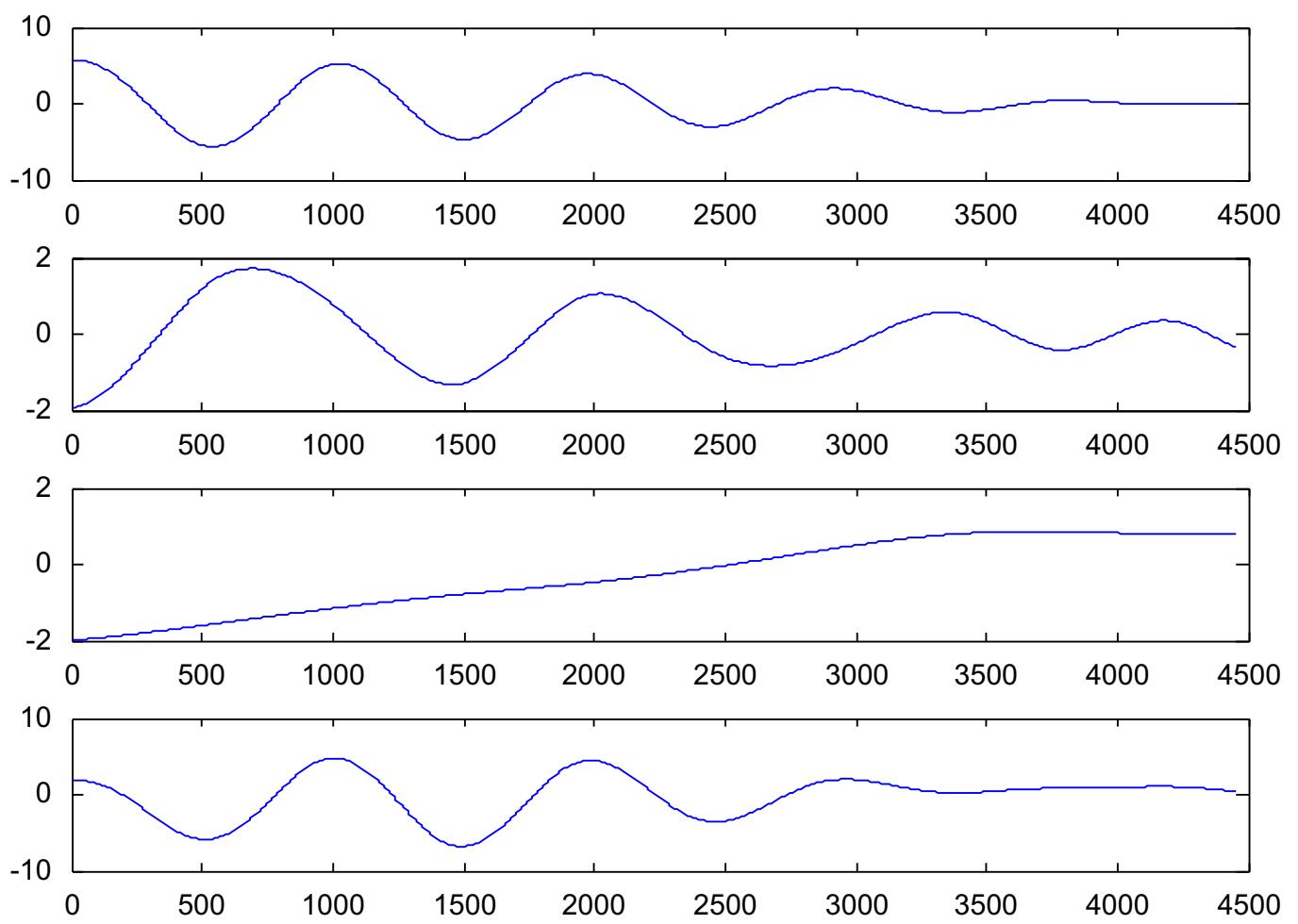

b
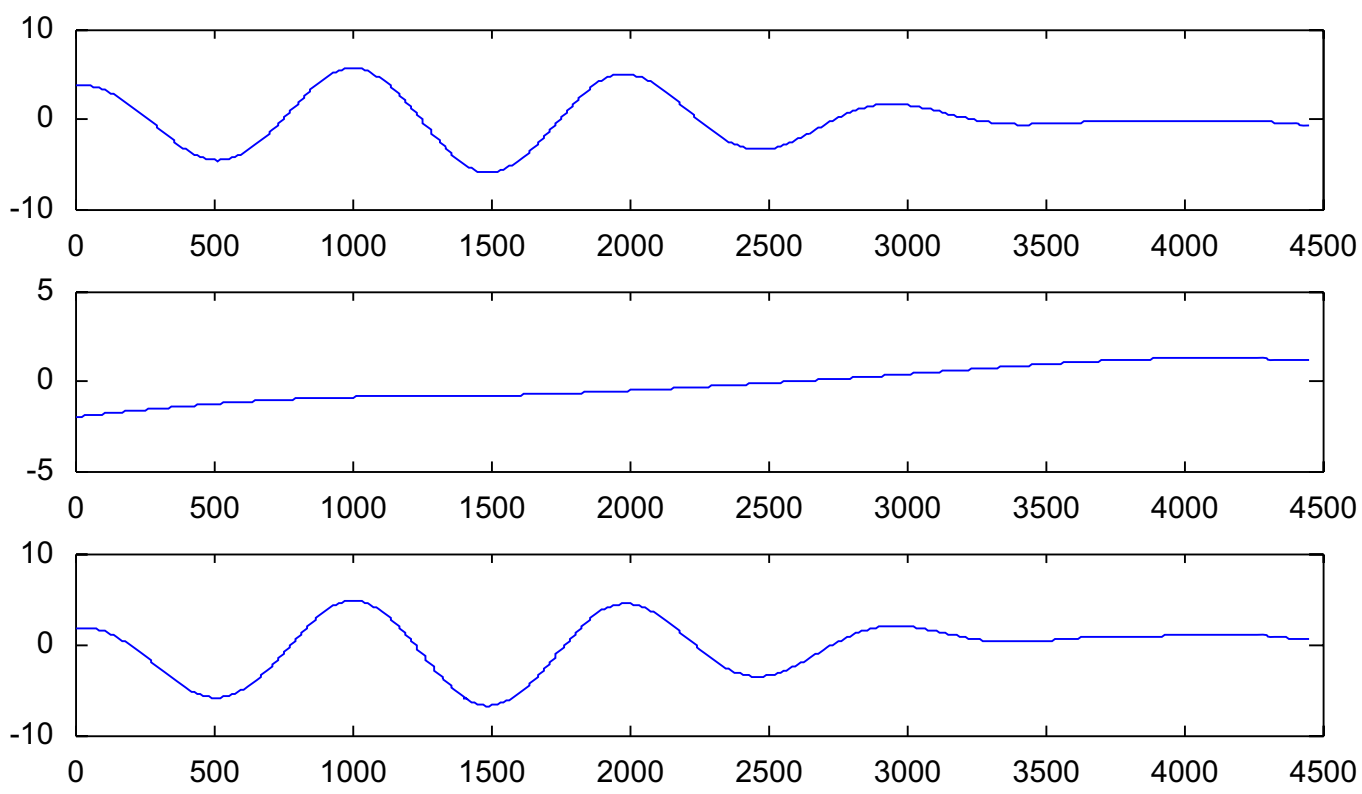

Fig. 1. The end effects: (a) Rilling algorithm and (b) the algorithm proposed here. The last strip represents the original signal.

to perform a side by side analysis. There is interdependence between the number of IMFs and resolution. When conducting signal analysis, if the resolution is fixed, the number of IMFs is allowed to vary from signal to signal. If the number of IMFs is to be fixed, then the resolution must be adjusted on a per signal basis.

\subsubsection{Flowchart of the algorithm}

The EMD algorithm proposed here is summarized in the flowchart shown in Fig. 2. 


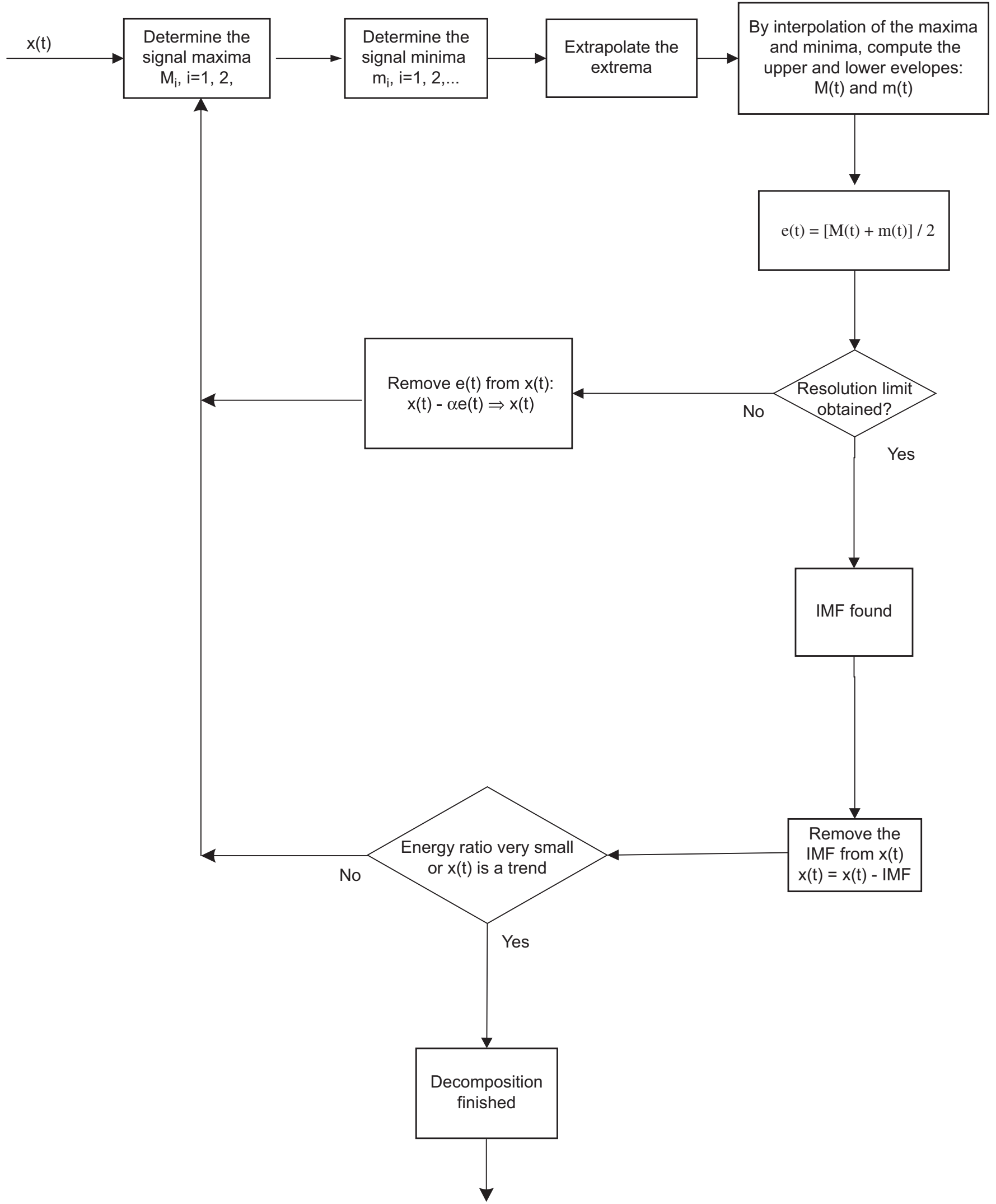

Several IMFs and a trend

Fig. 2. Flowchart of the proposed EMD algorithm. 


\section{On the Hilbert spectral analysis}

Spectral estimation is the second step of the HHT. This consists in computing the instantaneous amplitude and frequency for each IMF by using the HT and the analytic signal concept. This is another drawback of the HHT, because the HT uses the whole signal (theoretically from $-\infty$ to $+\infty$ ). As we have a finite segment of a signal, the window effect will distort its spectrum and as a consequence its HT. As we will show later, this can give poor frequency estimation. Besides, it is not easy to accept a global operator as base for a local estimation. On the other hand, we do not need to estimate an instantaneous amplitude, because we already have it, as we will see next.

\subsection{Demodulating the IMF}

Let $\varphi(t)$ be an IMF and $y(t)$ the corresponding analytic signal. So,

$$
\varphi(t)=\operatorname{Re}\left\{|y(t)| \mathrm{e}^{\operatorname{jarg}(y(t))}\right\}=|y(t)| \cos [\theta(t)],
$$

where $\theta(t)=\arg [y(t)]$. So, we obtained an the instantaneous amplitude and an oscillating function that is a constant AM/FM signal (not necessarily a sinusoid). If $|y(t)|$ is known, we can perform an amplitude demodulation and obtain

$$
s(t)=\cos [\theta(t)],
$$

such that

$$
|s(t)| \leqslant 1 .
$$

$s(t)$ can be considered as an FM signal. Its demodulation leads us to the instantaneous frequency. This will be considered in the next section. Now we will consider the amplitude demodulation.

At the end of the sifting procedure leading to the referred IMF, $\varphi(t)$, we also have its envelopes, $M(t)$ and $m(t)$. If these were the "true" envelopes, they would be symmetric and its difference would be the estimate of the amplitude modulating signal

$$
\varphi(t)=A(t) \cdot s(t)
$$

and

$$
A(t)=|y(t)|=M(t)-m(t) .
$$

As $M(t)$ and $m(t)$ are not truly symmetric, we must look for a more reliable estimate of $A(t)$. This can be achieved by the following procedure:

(a) Make $g(t)=|\varphi(t)|$.

(b) Compute the maxima of $g(t)$ and extrapolate them as described in Section 3.2.2.

(c) Interpolate those maxima to obtain an estimate of $A(t)$.

Now, it is enough to divide $\varphi(t)$ by $A(t)$ to obtain an FM signal, $s_{\mathrm{FM}}(t)$.

\subsection{On the instantaneous frequency}

Assume that the instantaneous frequency of $s_{\mathrm{FM}}(t)$ is a slowly time varying signal, so that we may consider it to be constant over small time intervals. Moreover, sample it to obtain a discrete-time signal that we can express in the format

$$
s_{\mathrm{FM}}(n) \approx \cos \left[2 \pi f\left(n_{0}\right) . n\right]
$$

valid for $n_{0}-N \leqslant n \leqslant n_{0}+N$. So, we assume that the frequency is constant in a window with length $2 N+1$, where $N$ is a positive integer. As shown in Appendix A, in this situation the instantaneous frequency can be 
obtained from

$$
\cos \left[2 \pi f\left(n_{0}\right)\right]=\frac{\sum_{2}^{L-1} s_{\mathrm{FM}}(n)\left[s_{\mathrm{FM}}(n-1)+s_{\mathrm{FM}}(n+1)\right]}{2 \sum_{2}^{L-1} s_{\mathrm{FM}}^{2}(n)},
$$

where $L$ is the number of available samples. In the case of a pure sinusoid this formula gives the correct value, provided we have at least three samples. For an FM signal we substitute $L=2 N+1$, as referred above. It defines the window of validity of the approximations. The choice of $N$ depends on the practical application. In the case of the chirp signal presented in Section 5.2.1, we used $N=10$. We have used values from 20 to 40 in EEG applications.

\section{Illustrating results}

\section{1. $E M D$}

\subsubsection{Adding two IMFs}

To illustrate the behaviour of the proposed algorithm and compare it with the original, we computed the corresponding decompositions for a signal that is a sum of two IMFs:

1. $x_{1}(n)=\sin \left(2 \pi f_{0} n\right)$,

2. $x_{2}(n)=2\left|\sin \left(2 \pi f_{1} n\right)\right|-1$

with $n=0,1, \ldots, 299, f_{1}=0.23 \mathrm{~Hz}$. We made two trials with $f_{0}=0.1$ and $0.3 \mathrm{~Hz}$. For comparison, we also present the results obtained with Rilling's routine [12]. In the following two figures, the signals in the upper two strips are the original signals. In Fig. 3, we present the results corresponding to the case of $f_{0}=0.1 \mathrm{~Hz}$. While our algorithm gives two IMFs, Rilling's gives six.

In Fig. 4 we show the results obtained with the same algorithms, but now with $f_{0}=0.3 \mathrm{~Hz}$.

Now the results are similar: both decompose into six IMFs.

\subsubsection{The chirp case}

Another signal naturally fitting the IMF definition is the constant amplitude chirp signal. As the sampling period is fixed and the chirp frequency is time dependent, there is no way of obtaining a sequence with all the maxima with the same amplitude. We present the example of the chirp signal: $\cos \left(2 \pi n^{2} / 4507+2 \pi n / 213\right)$, with $0 \leqslant n \leqslant 999$. The chirp rests undecomposed under our modified EMD, but under Rilling's EMD, a set of 10 IMFs is released as illustrated in Fig. 5. This is a non-desirable feature, because a signal already fitting the IMF definition should not be further decomposable.

\subsubsection{A robot movement signal}

In the first strip of Fig. 6, we present the $Y$ component of a horizontal movement of a robot and the corresponding EMD obtained with our and Rilling's algorithms. Again, the results with our algorithm seem more plausible.

\subsection{Frequency estimation}

Each IMF appears as an AM/FM modulated signal. In Huang et al. papers [1,2] a Hilbert spectral estimation is used to estimate the instantaneous frequency. As said before, we suggest here the use of a local AR approximation to estimate the frequency through the first reflection coefficient.

\subsubsection{Chirp signal}

In Fig. 7 we present illustrations of the frequency estimation for one chirp noisy signal.

As it can be seen, the AR method outperforms the HT estimation. 
a
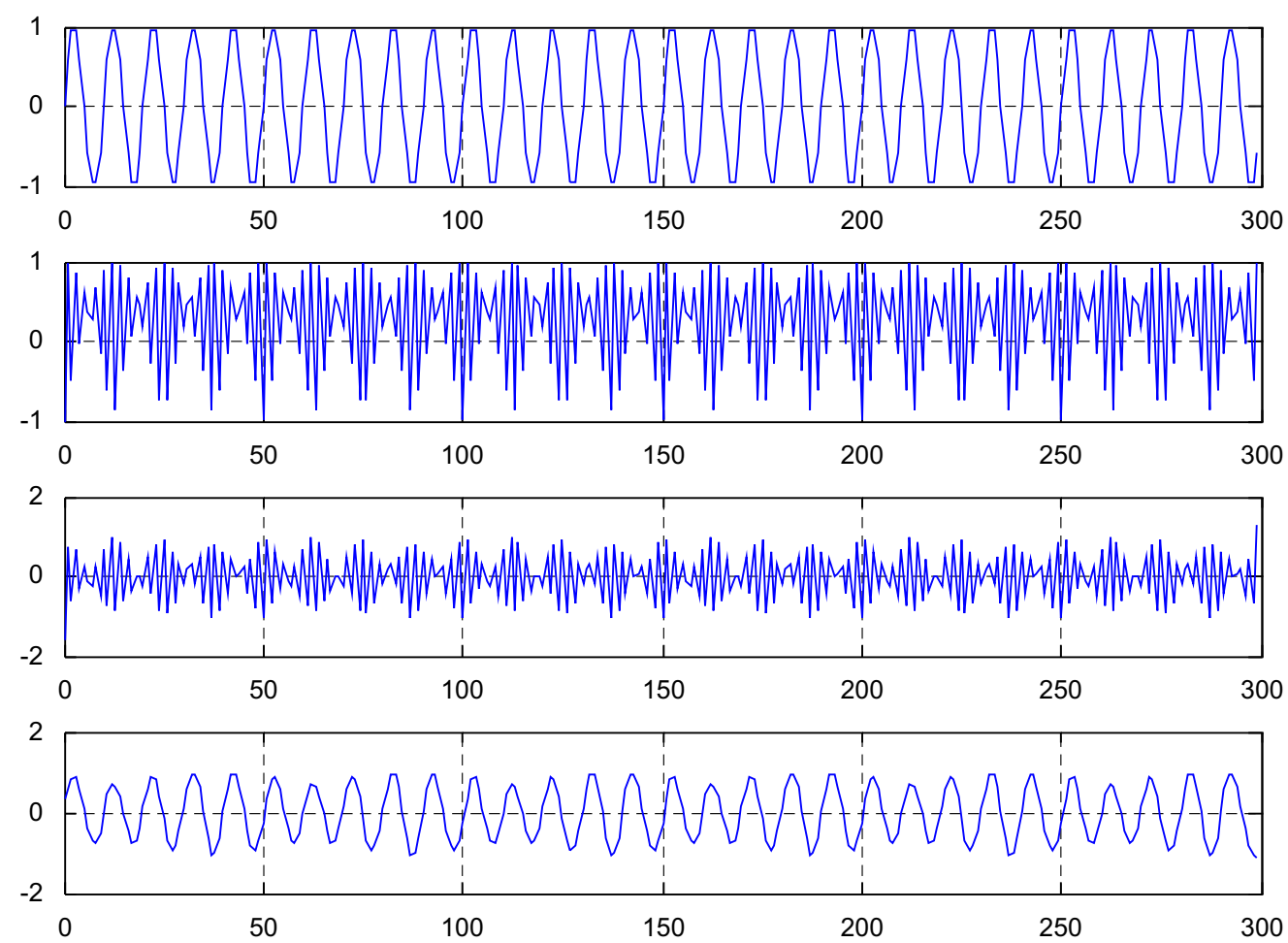

b

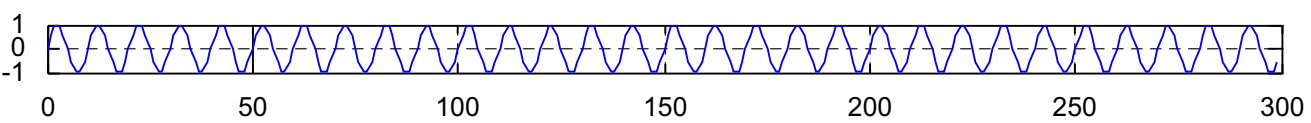

${ }_{-1}^{1}$

$\begin{array}{lllllll}0 & 50 & 100 & 150 & 200 & 250 & 300\end{array}$

$0_{-2}^{2}$ ${ }_{0}^{2}$

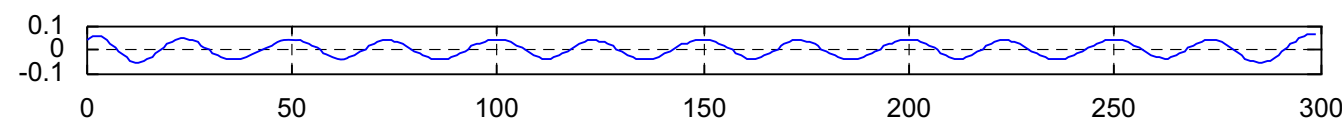

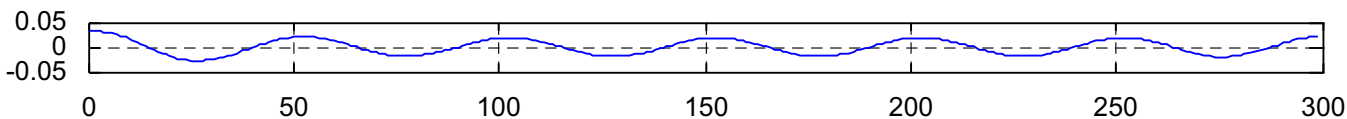

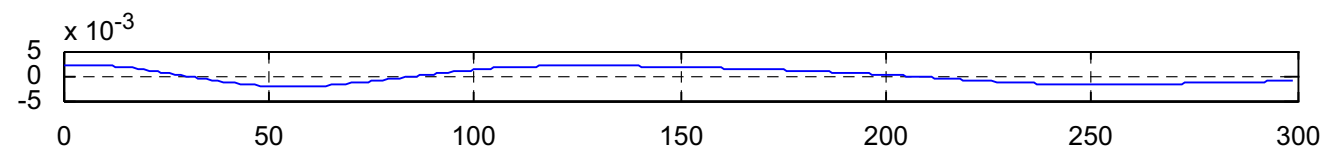

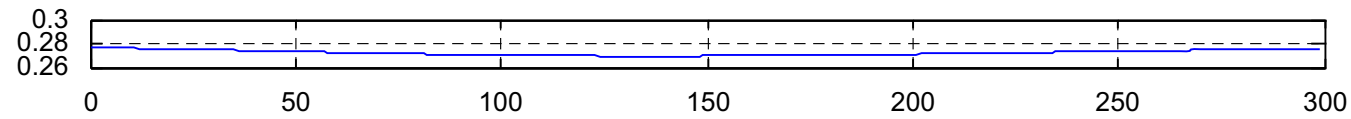

Fig. 3. Decomposition of the signal $\sin \left(2 \pi f_{0} n\right)+2\left|\sin \left(2 \pi f_{1} n\right)\right|-1$ using our and Rilling's algorithms with $f_{0}=0.1 \mathrm{~Hz}$ and $f_{1}=0.23 \mathrm{~Hz}$. The uppermost two strips represent the original signals. 
a

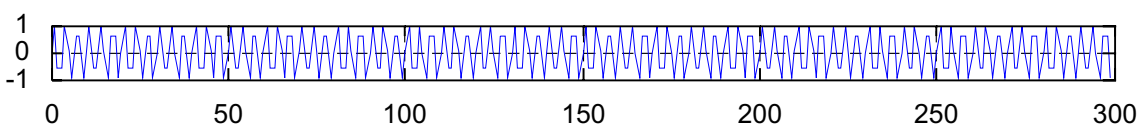

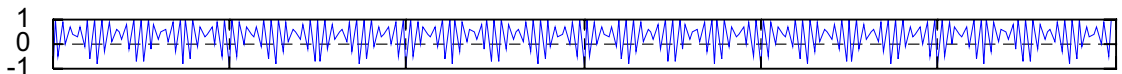

$\begin{array}{lllllll}0 & 50 & 100 & 150 & 200 & 250 & 300\end{array}$

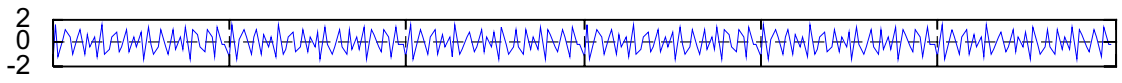

$\begin{array}{lllllll}0 & 50 & 100 & 150 & 200 & 250 & 300\end{array}$

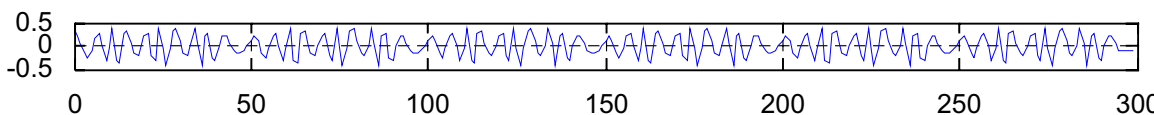

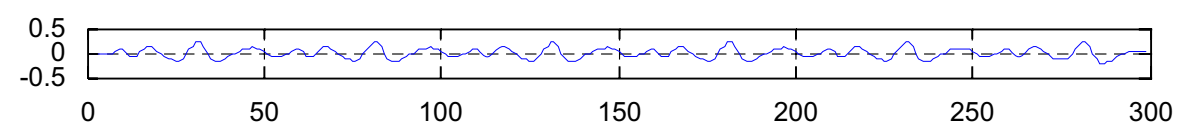
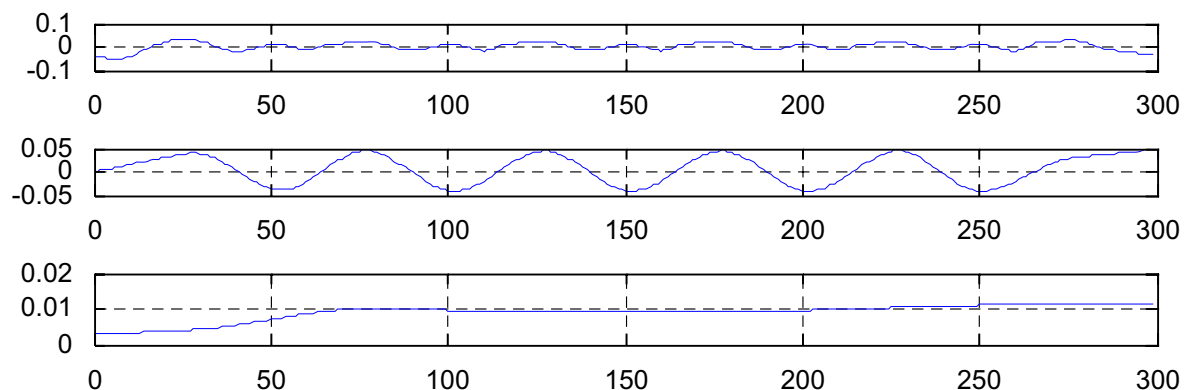

b

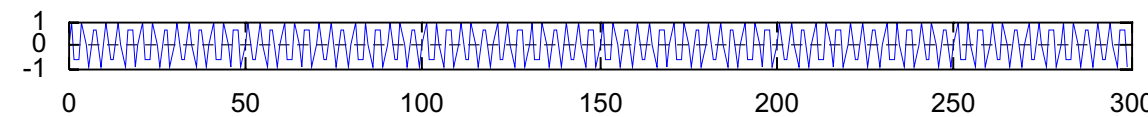

10

$\begin{array}{lllllll}0 & 50 & 100 & 150 & 200 & 250 & 300\end{array}$

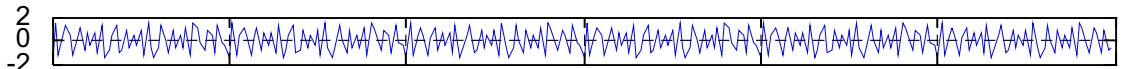

\begin{tabular}{|c|c|c|c|c|c|c|}
\hline 0 & 50 & 100 & 150 & 200 & 250 & 300 \\
\hline & $+A A$ & $A \wedge A \forall$ & $\forall A A$ & $7+A$ & 4 & \\
\hline & 50 & 100 & 150 & 200 & 250 & 300 \\
\hline
\end{tabular}

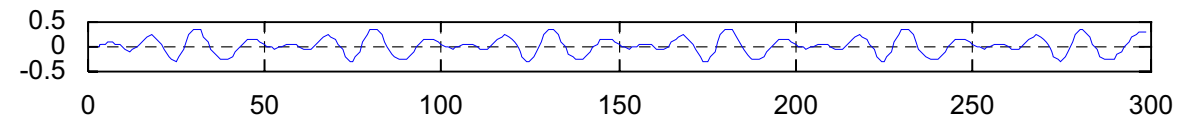

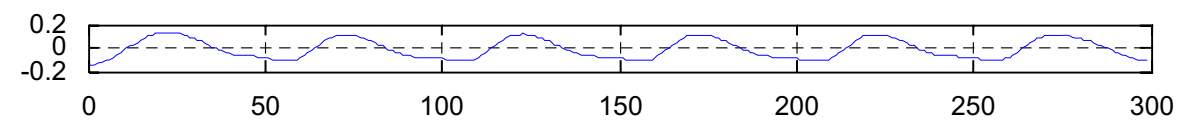

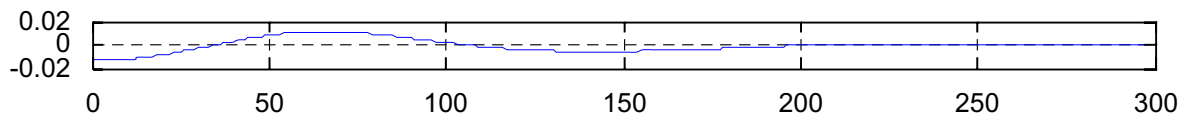

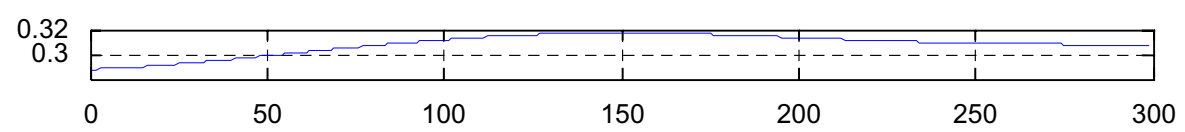

Fig. 4. Decomposition of the signal $\sin \left(2 \pi f_{0} n\right)+2\left|\sin \left(2 \pi f_{1} n\right)\right|-1$ using our and Rilling's algorithms with $f_{0}=0.3 \mathrm{~Hz}$ and $f_{1}=0.23 \mathrm{~Hz}$. The uppermost two strips represent the original signals. 

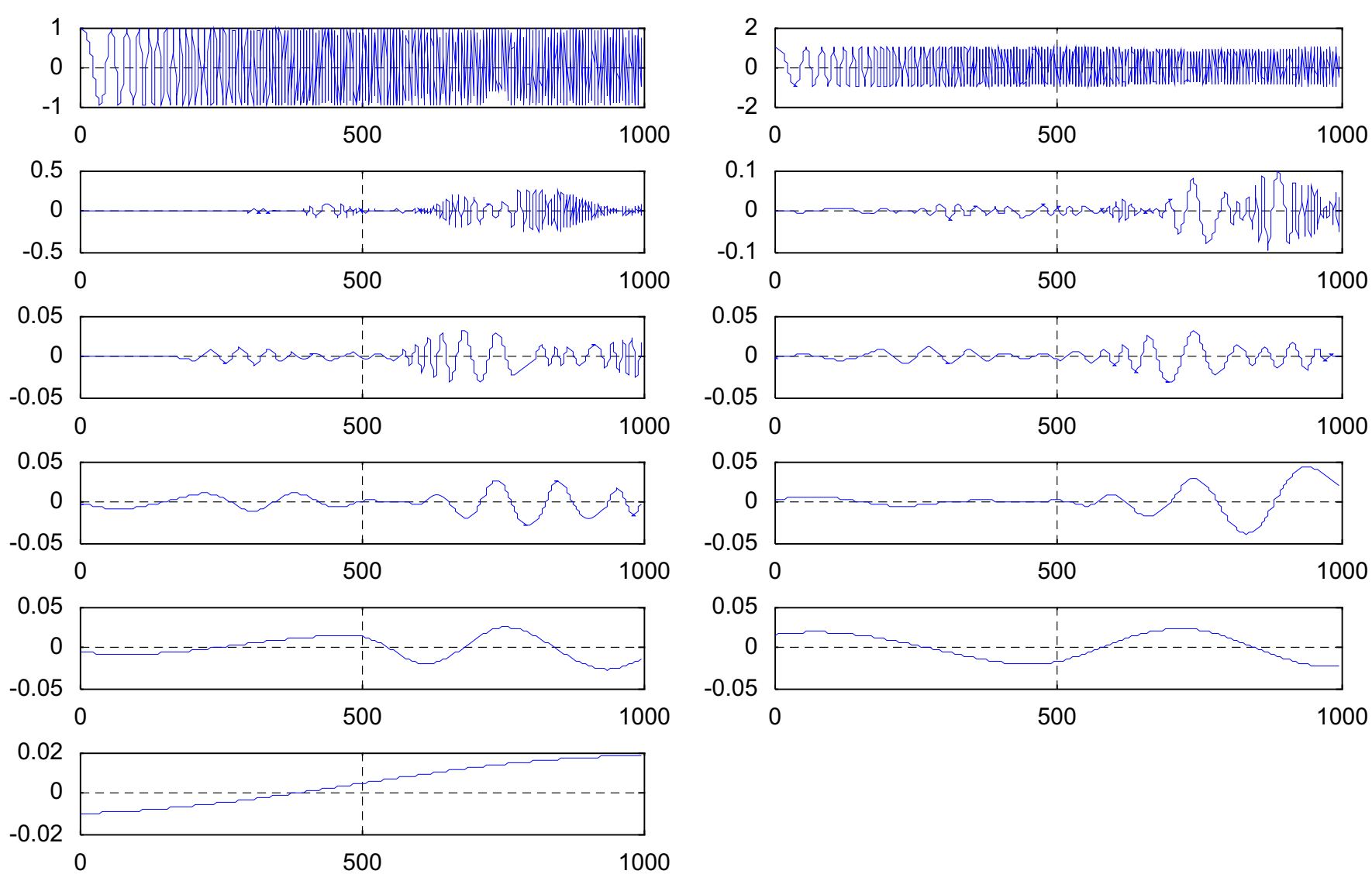

Fig. 5. Original Rilling's EMD of chirp signal $\cos \pi n^{2} / 4507+2 \pi n / 213$, with $0 \leqslant n \leqslant 999$. The interaction between the extrema locations and the sampling moments resulted in an enlarged IMF set.

\subsubsection{An ECG signal}

In the following we will present the results concerning the analysis of a segment of an ECG signal with five beats. In Fig. 8 we present the original signal and the corresponding IMFs obtained with the algorithm we propose here.

For comparison we are going to present the results of the time-frequency analysis, using two algorithms:

- Ours, that consists in the IMF amplitude demodulation followed by a frequency estimation using the AR modelling.

- Huang's approach based on the HT.

The energy operator demodulation proposed by Junsheng et al. [6] is not suitable to be used with short length signals since it cannot assure that the energy operator assumes only non-negative values.

As expected from our considerations in Section 4.1 the amplitudes are not very different (Fig. 9). This does not happen with the frequency estimates. It is clear that our approach gives more realistic and convincing results, mainly in the frequency computation (Fig. 10).

\subsubsection{The temperature in a dryer}

In Fig. 11 we present the time evolution of the temperature inside a dryer and its EMD using ours and Rilling's algorithms.

Similar to the last example, we computed the amplitudes and frequencies for both the decompositions. In Fig. 12 we present the results in a time-frequency plot. It seems clear that our algorithm seems to describe better the behaviour of the signal.

It is clear that the results stated in Fig. 12a seem more plausible when we look at the signal we are studying. 
a
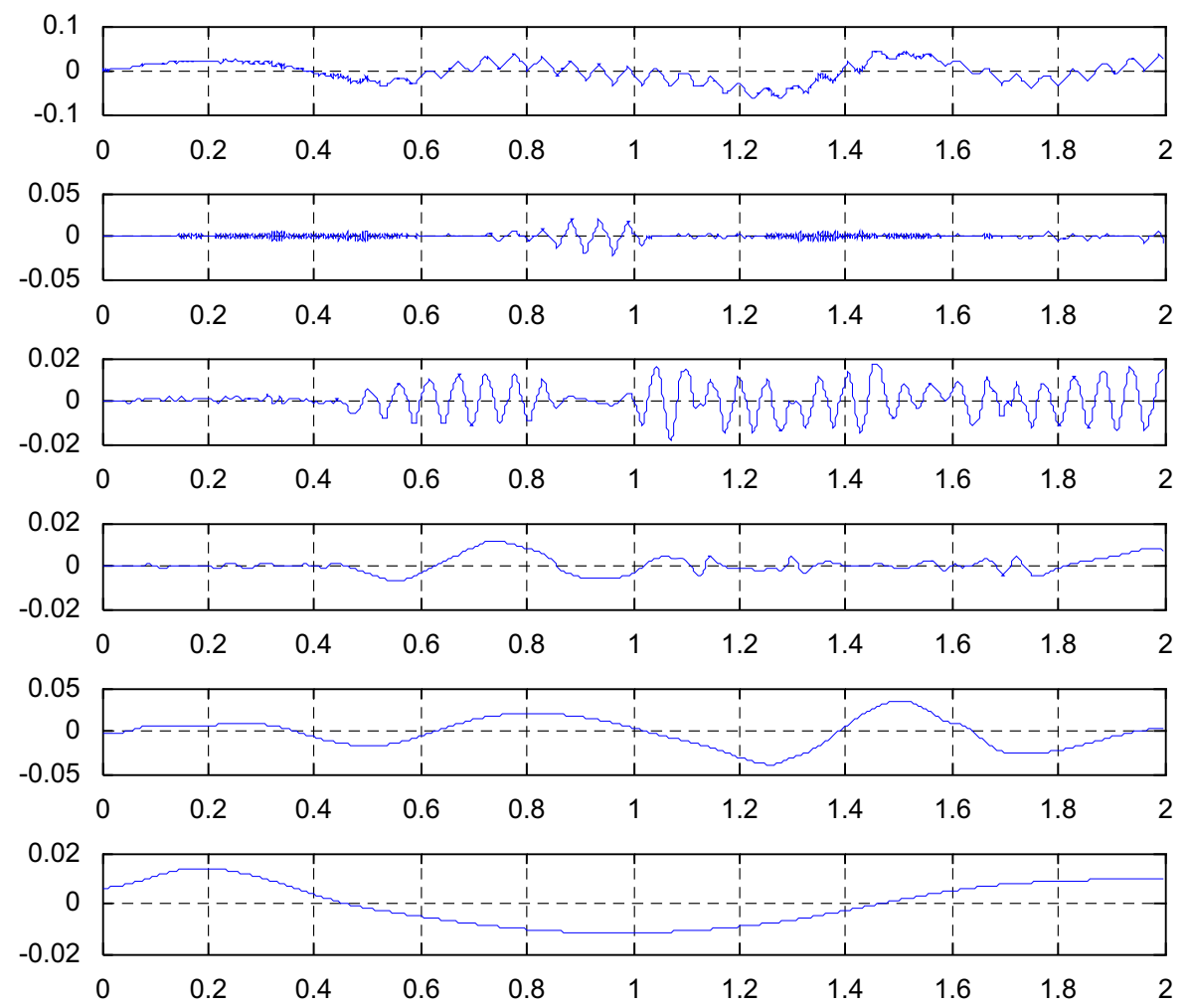

b
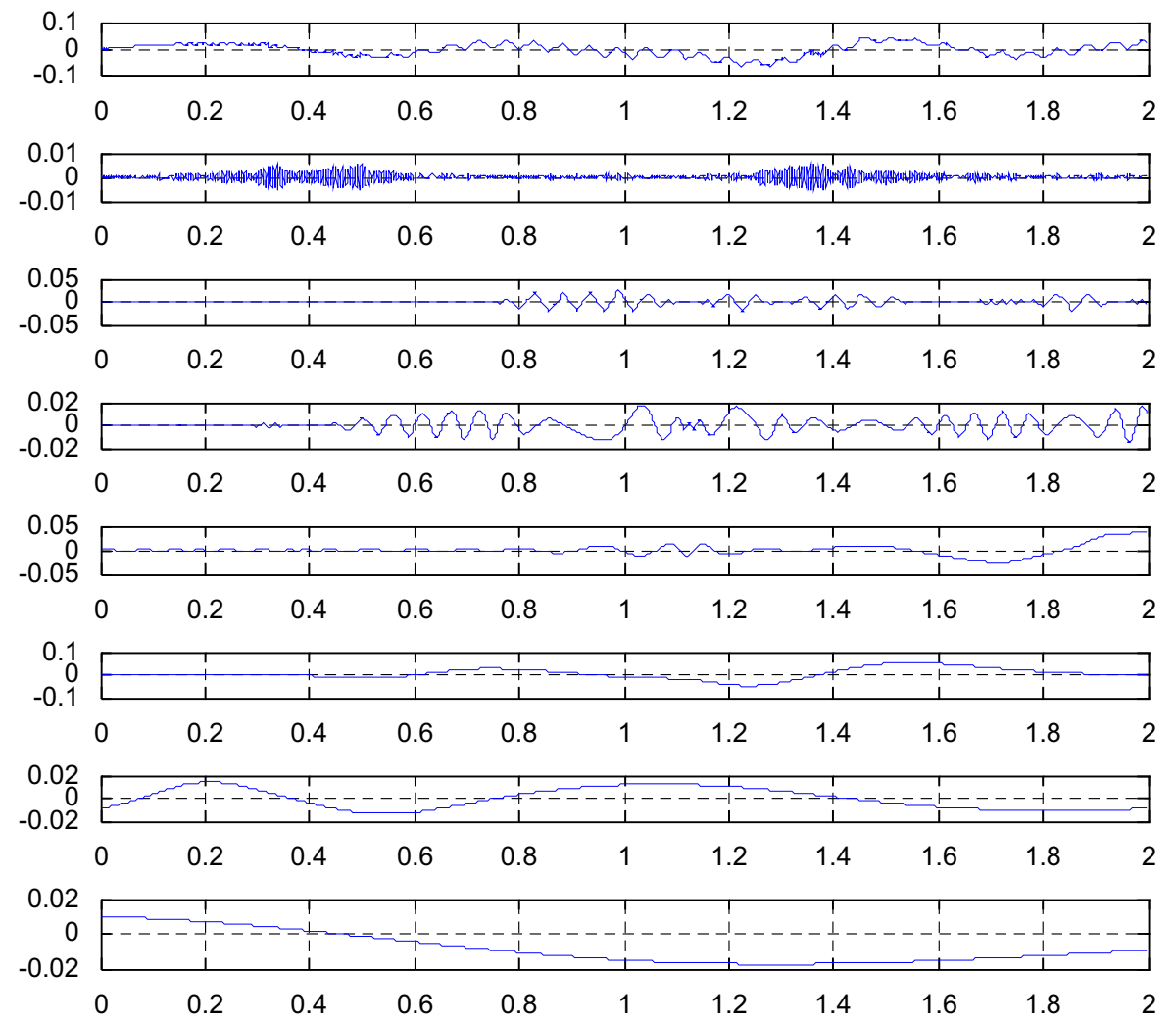

Fig. 6. EMD of $Y$ component of a horizontal movement of a robot, obtained from our (a) and Rilling's (b) EMD algorithms. 
a

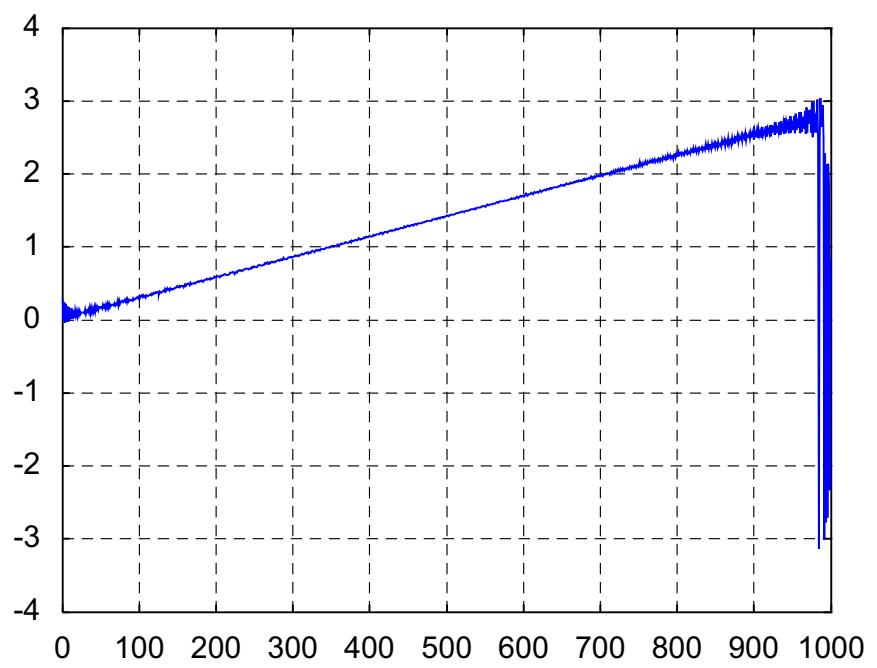

b

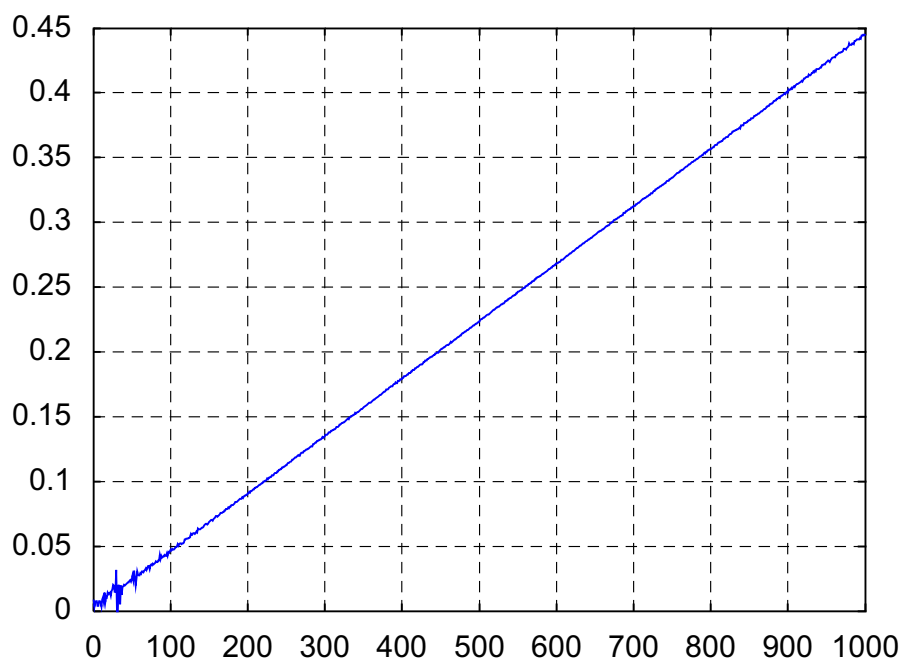

Fig. 7. Estimation of the instantaneous frequency with Hilbert transform (a) and AR methods (b).
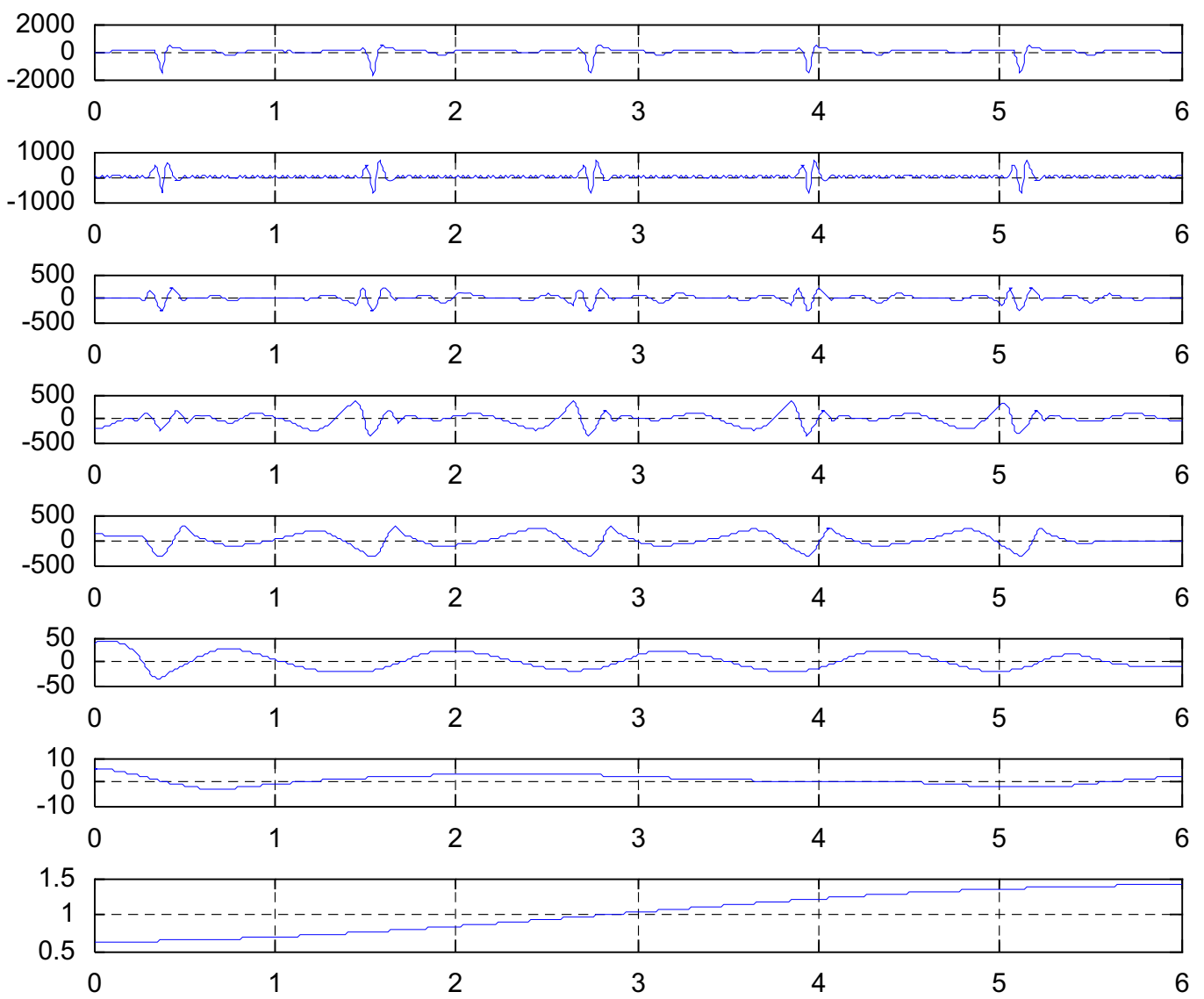

Fig. 8. EMD of a segment of an ECG. The original signal is in the upper strip.

\subsection{A brief comparison}

The above results show that:

- Our algorithm gives a small number of IMFs.

- Even at a glance, they seem to be more plausible as it is clear in the dryer case. 
a
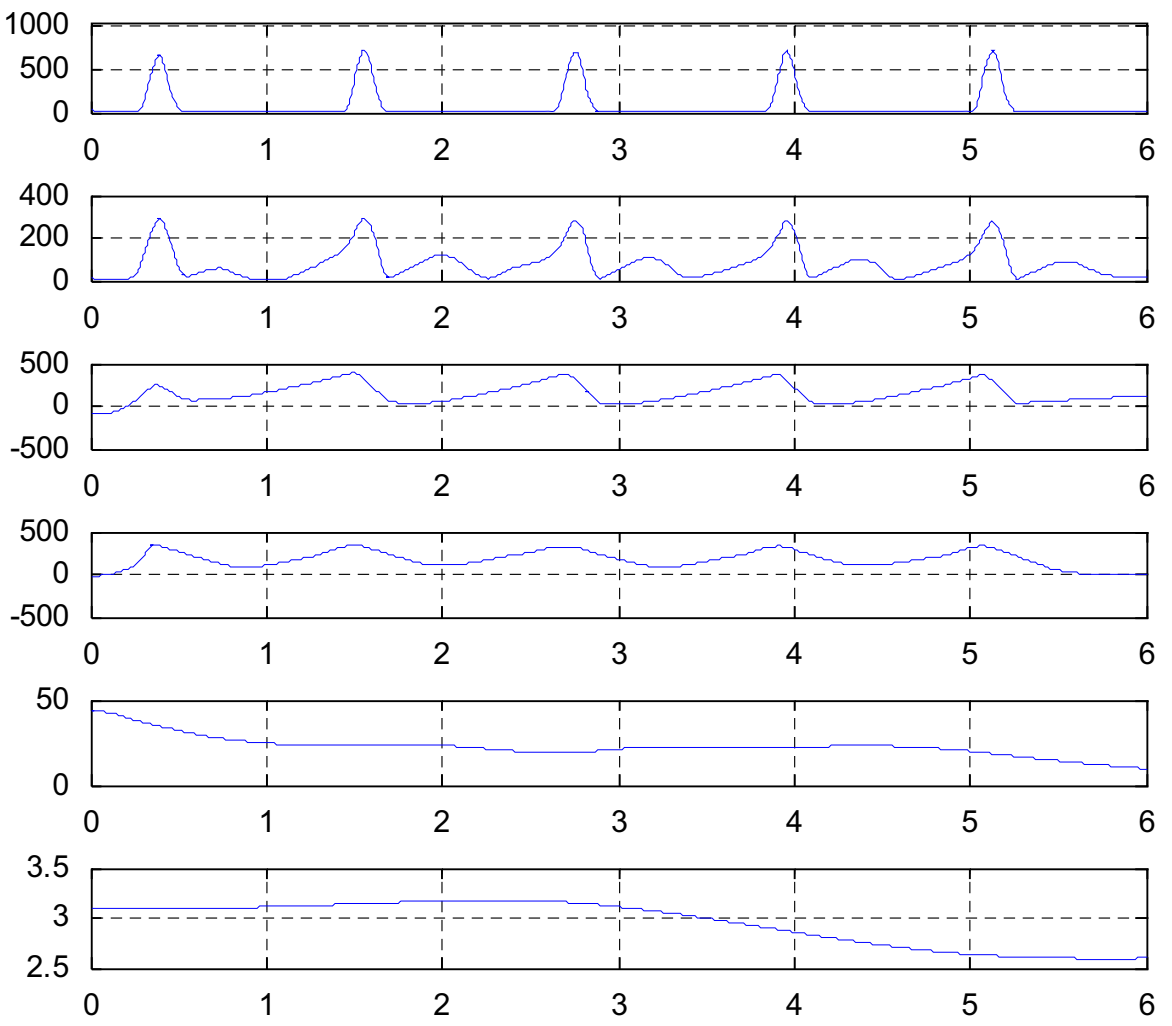

b
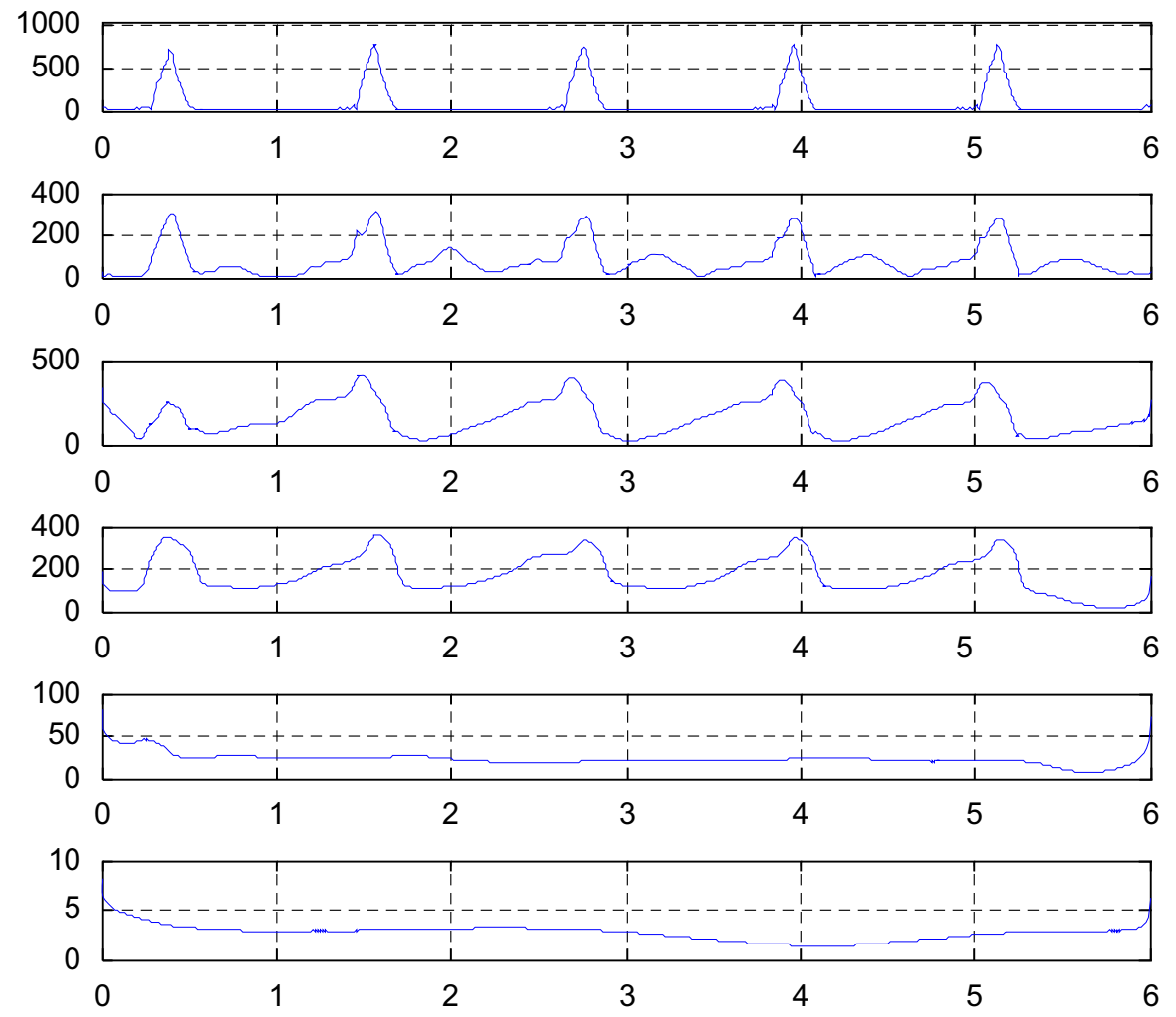

Fig. 9. Estimation of the amplitudes by AR modelling (a) and Hilbert transform (b). 
a
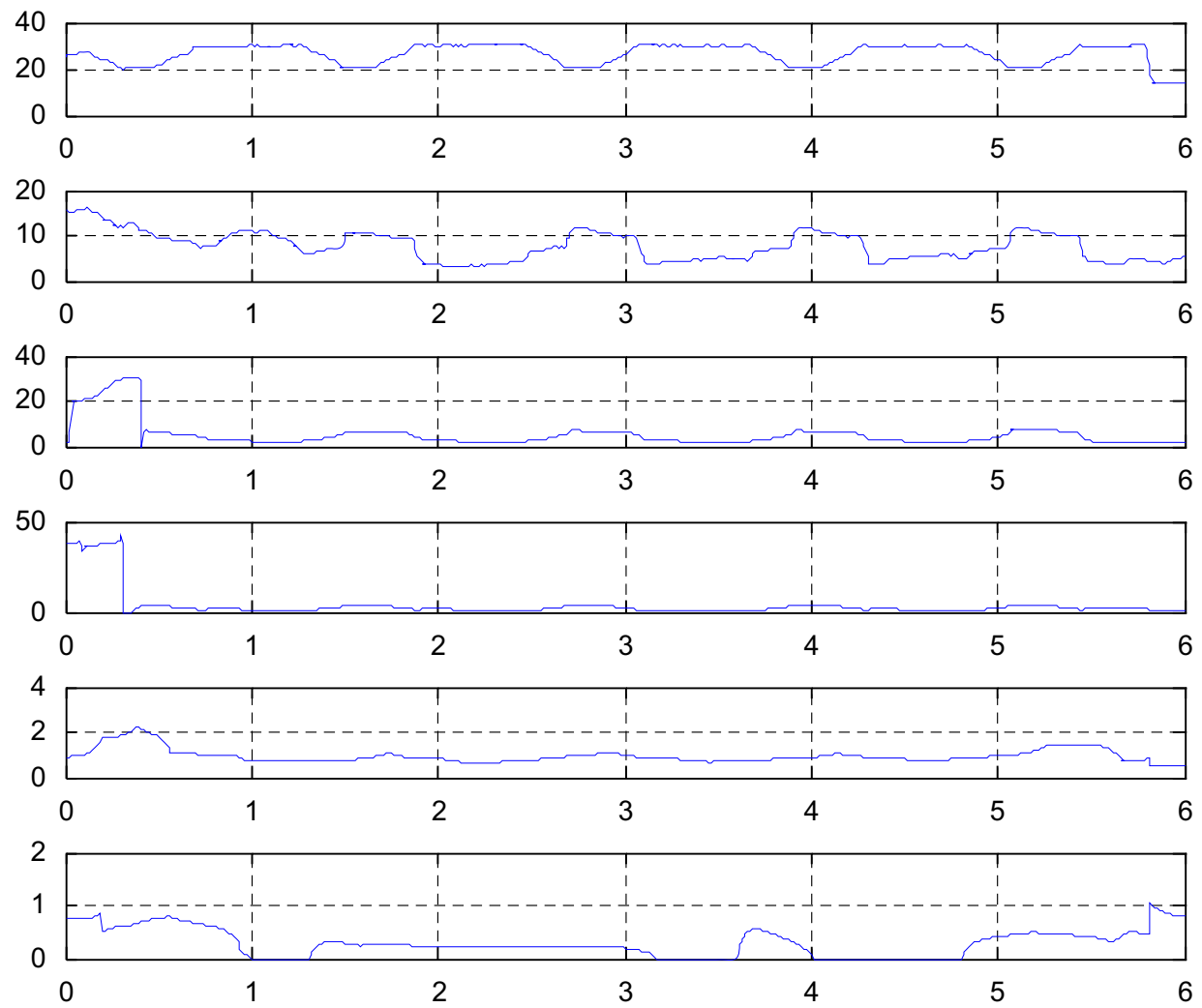

b
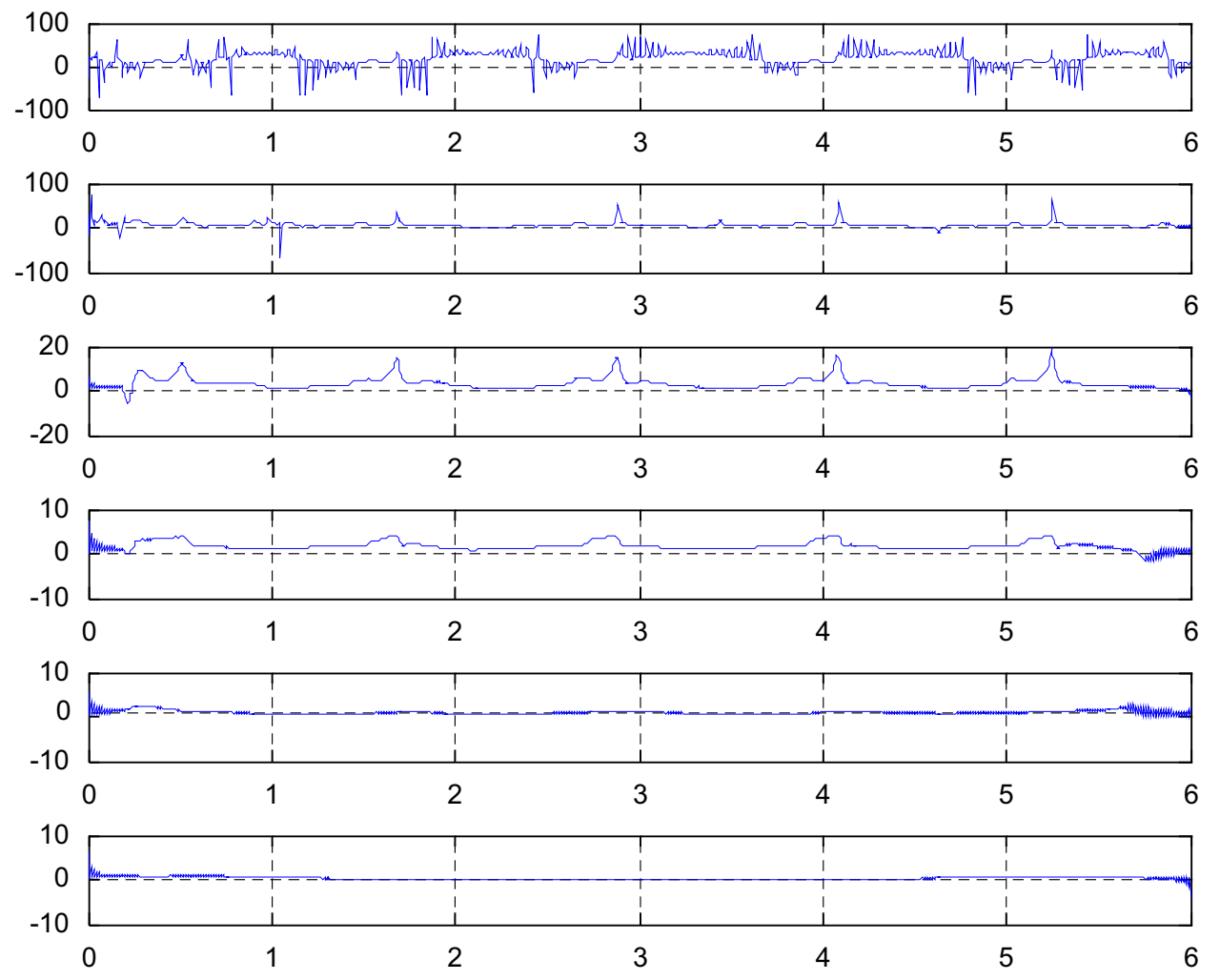

Fig. 10. Estimation of the frequencies by AR modelling (a) and Hilbert transform (b). 
a
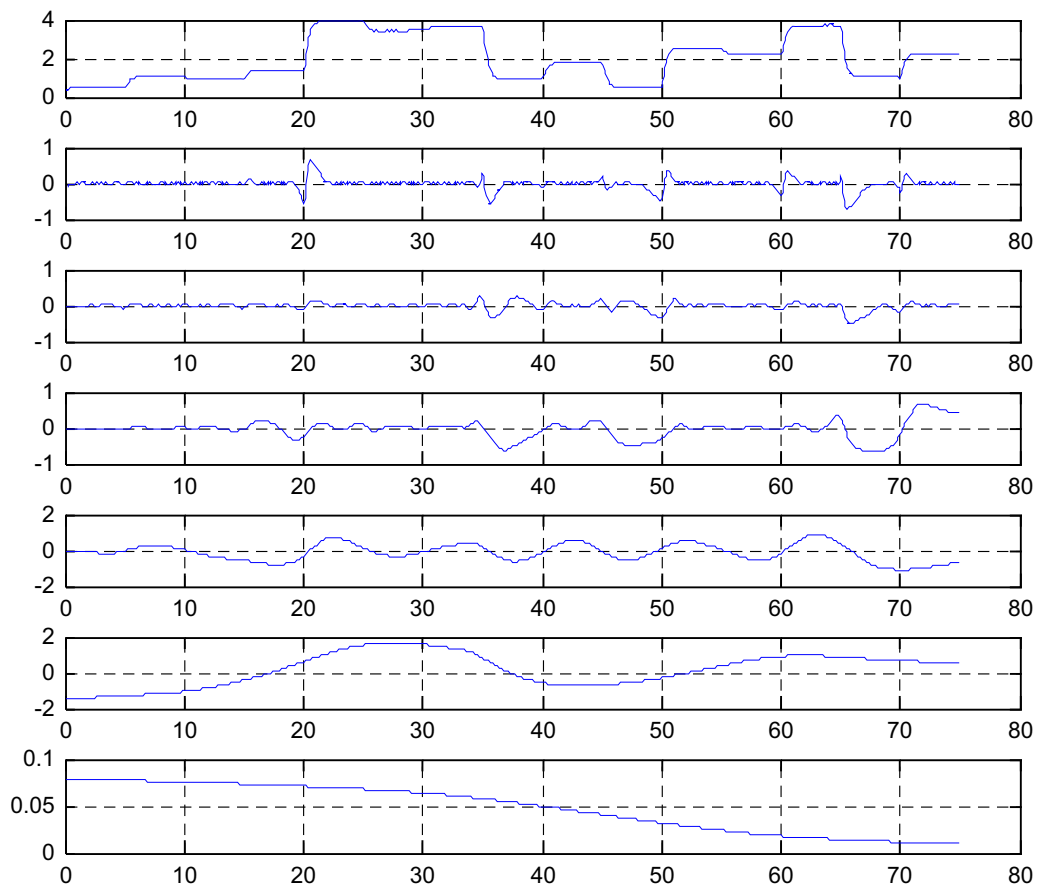

b

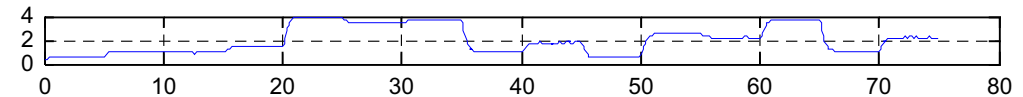

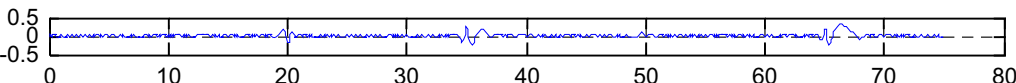

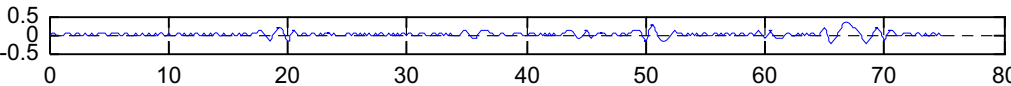

0.5
-0.5
0

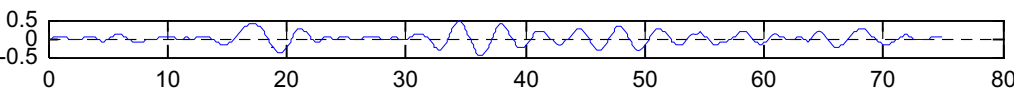

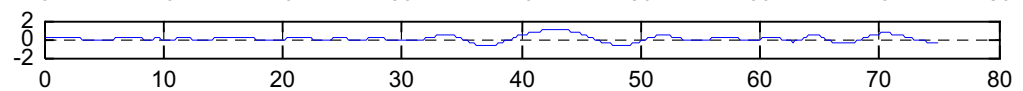

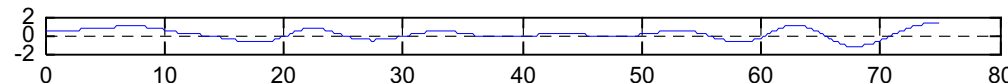
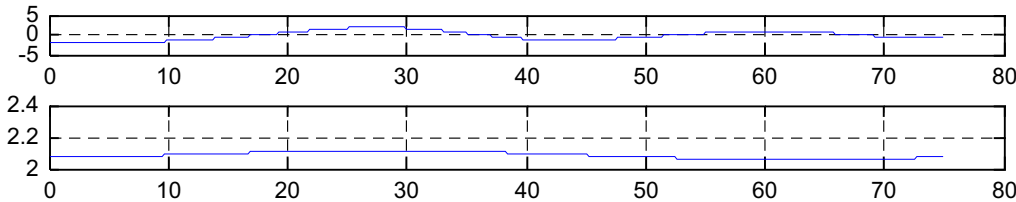

Fig. 11. The time evolution of the temperature inside a dryer and the corresponding EMD using our (a) and Rilling's (b) algorithms. The uppermost strip represents the temperature signal.

- The end effects are clearly less evident in our approach.

- The demodulation approach clearly outperforms Hilbert approach.

\section{Conclusions}

The empirical mode decomposition as a data driven alternative approach to the analysis of non-stationary signals was described. We studied it and revealed its features and some drawbacks. Here we proposed 
a

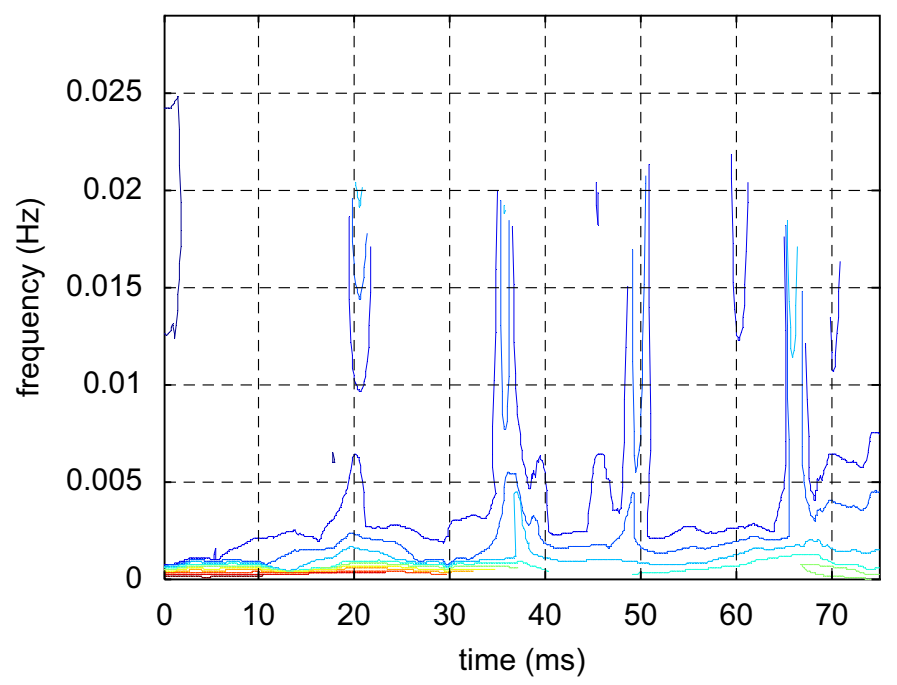

b

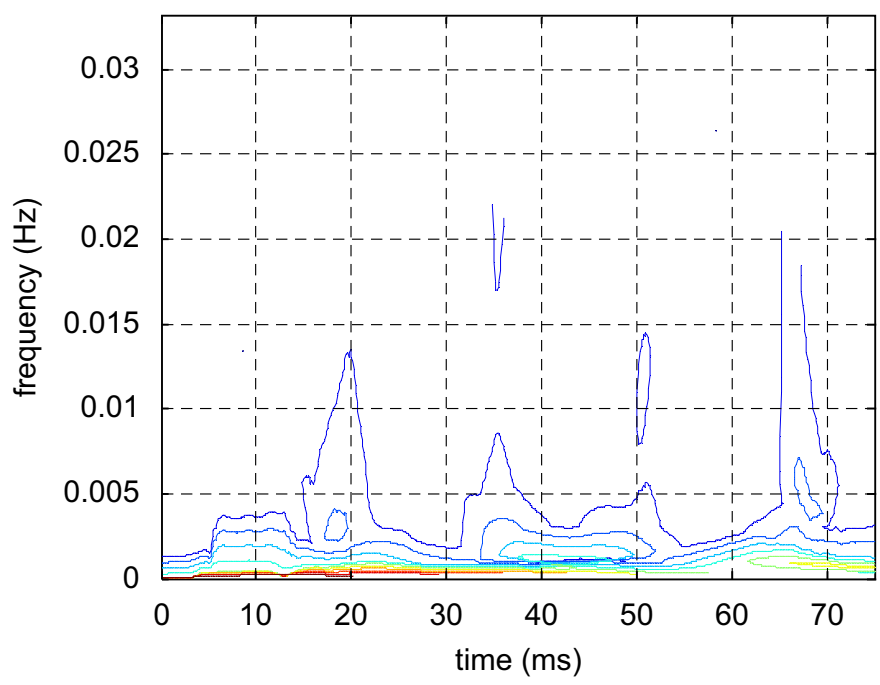

Fig. 12. The time-frequency plots for evolution of the temperature inside a dryer obtained from our (a) and Rilling's (b) EMD algorithms and using our demodulation procedure.

solutions for such difficulties in order to obtain an algorithm with better performances. We also criticized the use of the Hilbert transform for spectral estimation, presenting an alternative based on an AR local approximation. We also presented some simulation results.

\section{Appendix A}

Consider the signal $s_{\mathrm{FM}}(t)$ introduced in Section 4.1. Assume that its instantaneous frequency $f(t)$ is a slowly time varying signal, so that we may consider it to be constant in small intervals. We can write

$$
s_{\mathrm{FM}}(t) \approx \cos \left[2 \pi f\left(t_{0}\right) \cdot t\right]
$$

for $t$ in a small interval around $t_{0}$. Sampling this signal we can express it in the format

$$
s_{\mathrm{FM}}(n) \approx \cos \left[2 \pi f\left(n_{0}\right) \cdot n\right]
$$

valid for $n_{0}-N \leqslant n_{0 \leqslant} n_{0}+N$. So, we assume that the frequency is constant in a window with length $2 N+1$. In practice, we used $N=15$. Here, we propose an instantaneous frequency estimator based on a local AR approximation. It is known that, if $x_{n}=\cos (2 \pi f n+\varphi)$, with $f \in(0,1 / 2]$ and $\varphi \in(-\pi, \pi]$, it verifies an $\mathrm{AR}$ equation. In fact, we have

$$
\cos [2 \pi f n+\varphi]=\cos [2 \pi f(n-1)+\varphi] \cos (2 \pi f)-\sin [2 \pi f(n-1)+\varphi] \sin (2 \pi f)
$$

and

$$
\cos [2 \pi f(n-2)+\varphi]=\cos [2 \pi f(n-1)+\varphi] \cos (2 \pi f)+\sin [2 \pi f(n-1)+\varphi] \sin (2 \pi f) .
$$

Adding both equations, we obtain

$$
\cos [2 \pi f n+\varphi]+\cos [2 \pi f(n-2)+\varphi]=2 \cos [2 \pi f(n-1)+\varphi] \cos (2 \pi f)
$$

and

$$
\cos [2 \pi f n+\varphi]-2 \cos [2 \pi f(n-1)+\varphi] \cos (2 \pi f)+\cos [2 \pi f(n-2)+\varphi]=0
$$

giving

$$
x(n)-2 \cos (2 \pi f) x(n-1)+x(n-2)=0
$$


that leads to an AR model with polynomial

$$
1-2 \cos (2 \pi f) z^{-1}+z^{-2} \text {. }
$$

This polynomial is obtained using the Levinson recursion (see below) with the reflection coefficients:

$$
C_{1}=-\cos (2 \pi f)
$$

and

$$
C_{2}=1 \text {. }
$$

So, computing the first reflection coefficient in a window centred in the reference sample, we can obtain an estimate of the frequency.

Eq. (A.3) is a special case of the AR model

$$
\sum_{i=0}^{N_{0}} a_{i} x(n-i)=v(n)
$$

where $v(n)$ is white noise and $a_{i}, i=0,1, \ldots, N_{0}$, with $a_{0}=1$ are the AR parameters. These can be computed from a set of reflection coefficients by means of the Levinson recursion (see below).

To compute the reflection coefficient estimates, we use a modified Burg method [10]. We are going to introduce it in the following steps. Let $x(n)$ be a discrete time signal. For $N=1, \ldots, N_{0}$.

1. Define the forward and backward prediction errors by

$$
f_{n}^{N}=\sum_{i=0}^{N} a_{i}^{N} x(n-i)
$$

and

$$
b_{n}^{N}=\sum_{i=0}^{N} a_{i}^{N} x(n-N-1+i)
$$

and the error prediction power by

$$
P_{N}=\frac{1}{2(L-N)} \sum_{N+1}^{L}\left[\left(f_{n}^{N}\right)^{2}+\left(b_{n}^{N}\right) 2\right] .
$$

2. Use the Levinson recursion [10]

$$
a_{i}^{N}=a_{i}^{N-1}+C_{N} a_{N-i}^{N-1}, \quad i=0, \ldots, N
$$

to obtain

$$
f_{n}^{N}=f_{n}^{N-1}+C_{N} b_{n}^{N-1}
$$

and

$$
b_{n}^{N}=b_{n-1}^{N-1}+C_{N} f_{n-1}^{N-1}
$$

with $f_{n}^{0}=x(n)$ and $b_{n}^{0}=x(n-1)$.

3. With the above recursions, transform $P_{N}$ into a function of a unique variable- the reflection coefficient $C_{N}$ :

$$
P_{N}=\frac{1}{2(L-N)} \sum_{N+1}^{L}\left[\left(f_{n}^{N-1}+C_{N} \cdot b_{n-1}^{N-1}\right)^{2}+\left(b_{n-1}^{N-1}+C_{N} \cdot f_{n}^{N-1}\right)^{2}\right] .
$$

4. Derive $P_{N}$ relative to $C_{N}$ and equate to zero to obtain

$$
C_{N}=-\frac{\sum_{N+2}^{L}\left[f_{n}^{N-1} b_{n}^{N-1}+f_{n-1}^{N-1} b_{n-1}^{N-1}\right]}{\sum_{N+2}^{L}\left[\left(f_{n-1}^{N-1}\right)^{2}+\left(b_{n}^{N-1}\right)^{2}\right]} .
$$

For $N=1$, we immediately obtain Eq. (9). 


\section{References}

[1] N.E. Huang, Z. Shen, S.R. Long, M.L. Wu, H.H. Shih, Q. Zheng, N.C. Yen, C.C. Tung, H.H. Liu, The empirical mode decomposition and Hilbert spectrum for nonlinear and non-stationary time series analysis, Proceedings of the Royal Society of London A 454 (1998) 903-995.

[2] G. Rilling, P. Flandrin, P. Gonçalves, On empirical mode decomposition and its algorithms, in: IEEE-EURASIP Workshop on Nonlinear Signal and Image Processing NSIP-03, Grado (I), 2003.

[3] P. Flandrin, G. Rilling, P. Gonçalves, On empirical mode decomposition as a filter bank, IEEE Signal Processing Letters 11 (2) (2004).

[4] G. Rilling, P. Flandrin, On the influence of sampling on the empirical mode decomposition, in: Proceedings of the International Conference on Acoustics, Speech and Signal Processing, ICASSP 2006, pp. III-444-III-447.

[5] C. Junsheng, Y. Dejie, Y. Yu, Research on intrinsic mode function (IMF) criterion in EMD method, Mechanical Systems and Signal Processing 20 (2006) 817-824.

[6] C. Junsheng, Y. Dejie, Y. Yu, The application of the energy operator demodulation approach based on EMD in machinery fault diagnosis, Mechanical Systems and Signal Processing 21 (2007) 668-677.

[7] P. Maragos, J.F. Kaiser, T.F. Quatieri, On amplitude and frequency demodulation using energy operators, IEEE Transactions on Signal Processing 41 (4) (1993).

[8] S.R. Qin, Y.M. Zhong, A new envelope algorithm of Hilbert-Huang transform, Mechanical Systems and Signal Processing 20 (2006) 1941-1952.

[9] R.T. Rato, M.D. Ortigueira, A modified EMD algorithm for application in biomedical signal processing, in: Proceedings of the International Conference on Computational Intelligence in Medicine and Healthcare, CIMED 2005, Costa da Caparica, June 29-July 1, 2005 (CD edition).

[10] M.D. Ortigueira, J.M. Tribolet, Global versus local minimization in least-squares AR spectral estimation, Signal Processing 7 (3) (1984) 267-281.

[11] A.B. Carlson, Communication Systems, third ed., McGraw-Hill, New York, 1986.

[12] 〈http://perso.ens-lyon.fr/patrick.flandrin/emd.html〉. 